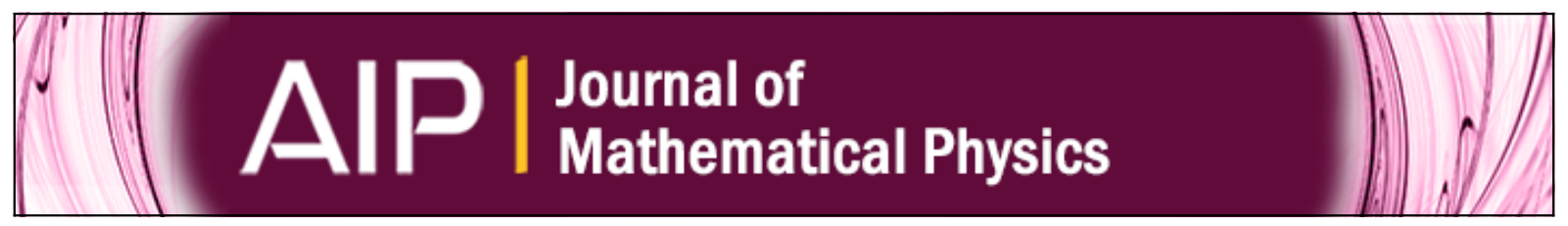

Generalized sine-Gordon/massive Thirring models and soliton/particle correspondences

José Acosta and Harold Blas

Citation: Journal of Mathematical Physics 43, 1916 (2002); doi: 10.1063/1.1454186

View online: http://dx.doi.org/10.1063/1.1454186

View Table of Contents: http://scitation.aip.org/content/aip/journal/jmp/43/4?ver=pdfcov

Published by the AIP Publishing

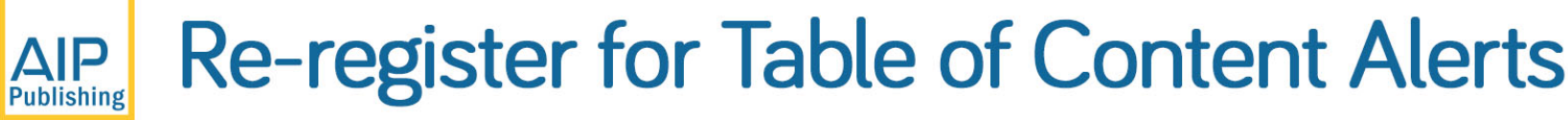




\title{
Generalized sine-Gordon/massive Thirring models and soliton/particle correspondences
}

\author{
José Acosta and Harold Blas a) \\ Instituto de Física Teórica, Universidade Estadual Paulista, Rua Pamplona 145, \\ 01405-900-São Paulo, S.P., Brazil
}

(Received 25 September 2001; accepted for publication 8 January 2002)

\begin{abstract}
We consider a real Lagrangian off-critical submodel describing the soliton sector of the so-called conformal affine $\operatorname{sl}(3)^{(1)}$ Toda model coupled to matter fields. The theory is treated as a constrained system in the context of Faddeev-Jackiw and the symplectic schemes. We exhibit the parent Lagrangian nature of the model from which generalizations of the sine-Gordon (GSG) or the massive Thirring (GMT) models are derivable. The dual description of the model is further emphasized by providing the relationships between bilinears of GMT spinors and relevant expressions of the GSG fields. In this way we exhibit the strong/weak coupling phases and the (generalized) soliton/particle correspondences of the model. The $\operatorname{sl}(n)^{(1)}$ case is also outlined. (C) 2002 American Institute of Physics.
\end{abstract}

[DOI: $10.1063 / 1.1454186]$

\section{INTRODUCTION}

Integrable theories in two dimensions have been an extraordinary laboratory for the understanding of basic nonperturbative aspects of physical theories and various aspects, relevant in more realistic four-dimensional models, have been tested. ${ }^{1}$ In particular the conformal affine Toda models coupled to (Dirac) matter fields $(\mathrm{CATM})^{2}$ for the $\operatorname{sl}(2)^{(1)}$ and $\operatorname{sl}(3)^{(1)}$ cases are discussed in Refs. 3, 4, and 5, respectively. The interest in such models comes from their integrability and duality properties, ${ }^{2,4}$ which can be used as toy models to understand some phenomena; such as a confinement mechanism in quantum chromodynamics $(\mathrm{QCD})^{3,5}$ and the electric-magnetic duality in four-dimensional gauge theories, conjectured in Ref. 6 and developed in Ref. 7. The affine Toda model coupled to matter field (ATM) type systems may also describe some low dimensional condensed matter phenomena, such as self-trapping of electrons into solitons, see, e.g., Ref. 8, tunneling in the integer quantum Hall effect, ${ }^{9}$ and, in particular, polyacteline molecule systems in connection with fermion number fractionization. ${ }^{10}$

Off-critical submodels, such as the $\operatorname{sl}(2)$ ATM, can be obtained at the classical or quantum mechanical level through some convenient reduction processes starting from CATM.,11 In the $\operatorname{sl}(2)$ case, using bosonization techniques, it has been shown that the classical equivalence between the $U(1)$ vector and topological currents holds true at the quantum level, and then leads to a bag model like mechanism for the confinement of the spinor fields inside the solitons; in addition, it has been shown that the $\operatorname{sl}(2)$ ATM theory decouples into a sine-Gordon model (SG) and a free scalar. ${ }^{3,12}$ These facts indicate the existence of a sort of duality in these models involving solitons and particles. ${ }^{6}$ The symplectic structure of the $s l(2)$ ATM model has recently been studied ${ }^{11}$ in the context of Faddeev-Jackiw $(\mathrm{FJ})^{13}$ and (constrained) symplectic methods. ${ }^{14,15}$ Imposing the equivalence between the $U(1)$ vector and topological currents as a constraint there have been obtained the SG or the massive Thirring (MT) model.

One of the difficulties with generalizations of complex affine Toda field theories, beyond $s u(2)$ and its associated SG model, has to do with unitarity. Whereas for practical applications

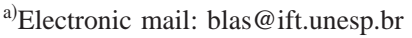


such as low dimensional condensed matter systems (see Ref. 16 and references therein) and $\mathrm{N}$-body problems in nuclear physics, ${ }^{17}$ the properties of interest are usually integrability and nonperturbative results of multifield Lagrangians. Therefore, integrable quantum field theories with several fields (bosons and/or fermions) are of some importance.

In this paper we construct many field generalizations of SG/MT models based on soliton/ particle duality and unitarity. Beyond the well-known $\operatorname{sl}(2)$ case the related $s l(n)^{(1)}$ CATM model does not possess a local Lagrangian, therefore we resort to an off-critical submodel Lagrangian with well behaved classical solutions making use of the results of Ref. 5. In Ref. 5 the authors studied the $\operatorname{sl}(3)^{(1)}$ CATM soliton solutions and some of their properties up to general twosoliton. Using the FJ and symplectic methods we show the parent Lagrangian ${ }^{18}$ nature of the $\operatorname{sl}(3)$ ATM model from which the generalized sine-Gordon (GSG) or the massive Thirring (GMT) models are derivable. We thus show that there are (at least classically) two equivalent descriptions of the model, by means of either the Dirac or the Toda type fields. The duality exchange of the coupling regimes $g \rightarrow 1 / g$ and the generalized soliton/particle correspondences in each $s l(2)$ ATM submodel will also be clear, which we uncover by providing explicit relationships between the GSG and GMT fields. We also outline the steps toward the $s l(n)$ affine Lie algebra generalizations. In this way we give a precise field content of both sectors; namely, the correct GMT/GSG duality, first undertaken in Ref. 19.

The paper is organized as follows. In Sec. II we define the $s$ (3) ATM model. Section III deals with the model in the FJ framework, the outcome is the GMT model. In Sec. IV, we attack the same problem from the point of view of symplectic quantization ${ }^{14,15}$ giving the Poisson brackets of the GMT and GSG models. Section V deals with the soliton/particle and strong/weak coupling correspondences. Section VI outlines the relevant steps toward the generalization to $\operatorname{sl}(n)$ ATM. In the appendix we present the construction of $s l(3)^{(1)}$ CATM model and its relationship to the (two-loop) Wess-Zumino-Novikov-Witten (WZNW) model.

\section{DESCRIPTION OF THE MODEL}

In affine Toda type theories the question of whether all mathematical solutions are physically acceptable deserves a careful analysis, especially if any consistent quantization of the models is discussed. The requirement of real energy density leads to certain reality conditions on the solutions of the model. In general, a few soliton solutions survive the reality constraint, if in addition one also demands positivity. These kind of issues are discussed in Refs. 20. Here we follow the prescription to restrict the model to a subspace of classical solutions which satisfy the physical principles of reality of energy density and soliton/particle correspondence.

In CATM models associated with the principal gradation of an affine Lie algebra we have a one-soliton solution (real Toda field) for each pair of Dirac fields $\psi^{i}$ and $\widetilde{\psi}^{i} .^{2}$ This fact allows us to make the identifications $\widetilde{\psi}^{i} \sim\left(\psi^{i}\right)^{*}$, and take real Toda fields. In the case of $\operatorname{sl}(2)^{(1)}$ CATM theory, this procedure does not spoil the particle-soliton correspondence. ${ }^{3,4}$

We consider the $\operatorname{sl}(3)^{(1)}$ CATM theory (see the Appendix) with the conformal symmetry gauge fixed ${ }^{21}$ by setting $\eta=0$ and the reality conditions

$$
\widetilde{\psi}^{j}=-\left(\psi^{j}\right)^{*}, \quad(j=1,2,3), \quad \varphi_{a}^{*}=\varphi_{a} \quad(a=1,2),
$$

or

$$
\begin{gathered}
\widetilde{\psi}^{j}=\left(\psi^{j}\right)^{*}, \quad j=1,2, \quad \widetilde{\psi}^{3}=-\left(\psi^{3}\right)^{*}, \\
\varphi_{1,2} \rightarrow \varphi_{1,2}-\pi \text { (the new } \varphi_{a} \text { 's being real fields), }
\end{gathered}
$$

where an asterisk means complex conjugation. The condition (2.2) must be supplied with $x^{\mu}$ $\rightarrow-x^{\mu}$. Moreover, for consistency of the equations of motion (A15)-(A23) under the reality conditions (2.1) and (2.2), from Eqs. (A16) to (A18), (A20), (A21), and (A23), we get the relationships 


$$
\widetilde{\psi}_{L}^{j} \psi_{R}^{3}-\widetilde{\psi}_{R}^{j} \psi_{L}^{3} e^{-3 i \varphi_{j}}=0, \quad j=1,2, \quad \psi_{L}^{1} \psi_{R}^{2} e^{-3 i \varphi_{1}}-\psi_{L}^{2} \psi_{R}^{1} e^{-3 i \varphi_{2}}=0 .
$$

Then, the above-given reality conditions and constraints allow us to define a suitable physical Lagrangian. Equations (A13), (A15)-(A23), supplied with (2.1) [or (2.2)] and (2.3), follow from the Lagrangian

$$
\frac{1}{k} \mathcal{L}=\sum_{j=1}^{3}\left[\frac{1}{24} \partial_{\mu} \phi_{j} \partial^{\mu} \phi_{j}+i \bar{\psi}^{j} \gamma^{\mu} \partial_{\mu} \psi^{j}-m_{\psi}^{j} \bar{\psi}^{j} e^{i \phi_{j} \gamma_{5}} \psi^{j}\right]
$$

where $\bar{\psi}^{j} \equiv\left(\psi^{j}\right)^{\dagger} \gamma_{0}, \phi_{1} \equiv 2 \varphi_{1}-\varphi_{2}, \phi_{2} \equiv 2 \varphi_{2}-\varphi_{1}, \phi_{3} \equiv \phi_{1}+\phi_{2}, m_{\psi}^{3}=m_{\psi}^{1}+m_{\psi}^{2}, k$ is an overall coupling constant and the $\varphi_{j}$ are real fields.

Equation (2.4) defines the sl(3) affine Toda theory coupled to matter fields (ATM). Notice that the space of solutions of $\operatorname{sl}(3)^{(1)}$ CATM model satisfying conditions (2.1)-(2.3) must be solutions of the $\operatorname{sl}(3)$ ATM theory (2.4). Indeed, it is easy to verify that the three species of one-soliton solutions $[S \equiv 1$-soliton $(\bar{S} \equiv 1$-antisoliton $)]:^{5} \quad\left\{\left(\varphi_{1}, \psi^{1}\right)_{S / \bar{S}}, \varphi_{2}=0, \psi^{2}=0, \psi^{3}=0\right\}$, $\left\{\left(\varphi_{2}, \psi^{2}\right)_{S / \bar{S}}, \varphi_{1}=0, \psi^{1}=0, \psi^{3}=0\right\}$, and $\left\{\left(\varphi_{1}+\varphi_{2}, \psi^{3}\right)_{S / \bar{S}}, \varphi_{1}=\varphi_{2}, \psi^{1}=0, \psi^{2}=0\right\}$ satisfy the equations of motion, i.e., each positive root of $\operatorname{sl}(3)$ reproduces the $s l(2)$ ATM case. ${ }^{3,4}$ Moreover, these solutions satisfy the above-given reality conditions and constriants (2.1)-(2.3) [with (2.1) and (2.2) for $S$ and $\bar{S}$, respectively], and the equivalence between the $U(1)$ vector and topological currents (A29). Then, the soliton/particle correspondences survive the above-given reduction processes performed to define the $s l(3)$ ATM theory.

The class of two-soliton solutions of $\operatorname{sl}(3)^{(1)} \mathrm{CATM}^{5}$ behave as follows: (i) they are given by six species associated with the pair $\left(\alpha_{i}, \alpha_{j}\right), i \leqslant j ; i, j=1,2,3$; where the $\alpha$ 's are the positive roots of $\operatorname{sl}(3)$ Lie algebra. Each species $\left(\alpha_{i}, \alpha_{i}\right)$ solves the $\operatorname{sl}(2)$ CATM submodel ${ }^{22}$ (ii) satisfy the $U(1)$ vector and topological currents equivalence (A29).

\section{THE GENERALIZED MASSIVE THIRRING MODEL (GMT)}

Let us consider the following Lagrangian:

$$
\frac{1}{k} \mathcal{L}=\sum_{j=1}^{3}\left[\frac{1}{24} \partial_{\mu} \phi_{j} \partial^{\mu} \phi_{j}+i \bar{\psi}^{j} \gamma^{\mu} \partial_{\mu} \psi^{j}-m_{\psi}^{j} \bar{\psi}^{j} e^{i \phi_{j} \gamma_{5}} \psi^{j}+\lambda_{\mu}^{j}\left(m^{j} \bar{\psi}^{j} \gamma^{\mu} \psi^{j}-\epsilon^{\mu \nu} \partial_{\nu}\left(q_{j} \phi_{j}\right)\right)\right],
$$

where the ATM Lagrangian (2.4) is supplied with the constraints, $\left(m^{l} \bar{\psi}^{l} \gamma^{\mu} \psi^{l}+\left(m^{3} / 2\right) \bar{\psi}^{3} \gamma^{\mu} \psi^{3}\right.$ $\left.-\epsilon^{\mu \nu} \partial_{\nu} \phi_{l}\right),(l=1,2)$, with the help of the Lagrange multipliers $\lambda_{\mu}^{j}\left(\lambda_{\mu}^{3} \equiv\left(\lambda_{\mu}^{1}+\lambda_{\mu}^{2}\right) / 2, q_{1} \equiv q_{2}\right.$ $\left.\equiv 1, q_{3} \equiv 0\right)$. Their total sum bears an intriguing resemblance to the $U(1)$ vector and topological currents equivalence (A29); however, the $m^{j}$,s here are some arbitrary parameters. The same procedure has been used, for example, to incorporate the left-moving condition in the study of chiral bosons in two dimensions. ${ }^{23}$ The constraints in (3.1) will break the left-right local symmetries (A25)-(A28) of $s l(3)$ ATM (2.4). In order to apply the FJ method we should write (3.1) in the first-order form in time derivative, so let us define the conjugated momenta

$$
\begin{gathered}
\pi_{1} \equiv \pi_{\phi_{1}}=\frac{1}{12}\left(2 \dot{\phi}_{1}+\dot{\phi}_{2}\right)+\lambda_{1}^{1}, \quad \pi_{2} \equiv \pi_{\phi_{2}}=\frac{1}{12}\left(2 \dot{\phi}_{2}+\dot{\phi}_{1}\right)+\lambda_{1}^{2}, \\
\pi_{\lambda_{\mu}^{1}}=0, \quad \pi_{\lambda_{\mu}^{2}}=0, \quad \pi_{R}^{j} \equiv \pi_{\psi_{R}}^{j}=-i \widetilde{\psi}_{R}^{j}, \quad \pi_{L}^{j} \equiv \pi_{\psi_{L}}^{j}=-i \widetilde{\psi}_{L}^{j} .
\end{gathered}
$$

We are assuming that Dirac fields are anticommuting Grasmannian variables and their momenta variables defined through left derivatives. Then, as usual, the Hamiltonian is defined by (sum over repeated indices is assumed)

$$
\mathcal{H}_{c}=\pi_{1} \dot{\phi}_{1}+\pi_{2} \dot{\phi}_{2}+\dot{\psi}_{R}^{j} \pi_{R}^{j}+\dot{\psi}_{L}^{j} \pi_{L}^{j}-\mathcal{L}
$$


Explicitly the Hamiltonian density becomes

$$
\begin{aligned}
\mathcal{H}_{c}= & 2\left(\pi_{j}\right)^{2}+4\left(\lambda_{1}^{1}\right)^{2}+4\left(\lambda_{1}^{2}\right)^{2}-\lambda_{1}^{1} \mathcal{J}_{1}-\lambda_{1}^{2} \mathcal{J}_{2}-4\left(\lambda_{1}^{1} \lambda_{1}^{2}\right)+\frac{1}{24}\left(\phi_{j, x}\right)^{2}-\pi_{R}^{j} \psi_{R, x}^{j}+\pi_{L}^{j} \psi_{L, x}^{j} \\
& +i m_{\psi}^{j}\left(e^{-\phi_{j}} \widetilde{\psi}_{R}^{j} \psi_{L}^{j}-e^{\phi_{j}} \widetilde{\psi}_{L}^{j} \psi_{R}^{j}\right)+\lambda_{0}^{1}\left[J_{1}^{0}-\phi_{1, x}\right]+\lambda_{0}^{2}\left[J_{2}^{0}-\phi_{2, x}\right],
\end{aligned}
$$

where $\pi_{3} \equiv \pi_{1}-\pi_{2}, \mathcal{J}_{1} \equiv J_{1}^{1}+4\left(2 \pi_{1}-\pi_{2}\right), \mathcal{J}_{2} \equiv J_{2}^{1}+4\left(2 \pi_{2}-\pi_{1}\right)$, and

$$
J_{1}^{\mu}=m^{1} j_{l}^{\mu}+\frac{m^{3}}{2} j_{3}^{\mu}, \quad J_{2}^{\mu}=m^{2} j_{2}^{\mu}+\frac{m^{3}}{2} j_{3}^{\mu}, \quad j_{l}^{\mu} \equiv \bar{\psi}^{l} \gamma^{\mu} \psi^{l}, \quad l=1,2,3 .
$$

Let us observe that each $U(1)$ Noether current of the $\operatorname{sl}(3)$ ATM theory defined in (2.4) is conserved separately, i.e., $\partial_{\mu} j_{l}^{\mu}=0, l=1,2,3$.

Next, the same Legendre transform (3.3) is used to write the first-order Lagrangian

$$
\mathcal{L}=\pi_{1} \dot{\phi}_{1}+\pi_{2} \dot{\phi}_{2}+\dot{\psi}_{R}^{j} \pi_{R}^{j}+\dot{\psi}_{L}^{j} \pi_{L}^{j}-\mathcal{H}_{c}
$$

Our starting point for the FJ analysis will be this first-order Lagrangian. Then the EulerLagrange equations for the Lagrange multipliers allow one to solve two of them

$$
\lambda_{1}^{1}=\frac{2 \mathcal{J}_{1}+\mathcal{J}_{2}}{12}, \quad \lambda_{1}^{2}=\frac{2 \mathcal{J}_{2}+\mathcal{J}_{1}}{12}
$$

and the remaining equations lead to two constraints,

$$
\Omega_{1} \equiv J_{1}^{0}-\phi_{1, x}=0, \quad \Omega_{2} \equiv J_{2}^{0}-\phi_{2, x}=0 .
$$

The Lagrange multipliers $\lambda_{1}^{1}$ and $\lambda_{1}^{2}$ must be replaced back in (3.6) and the constraints (3.8) solved. First, let us replace the $\lambda_{1}^{1}$ and $\lambda_{1}^{2}$ multipliers into $\mathcal{H}_{c}$, then one gets

$$
\begin{aligned}
\mathcal{H}_{c}^{\prime}= & 2\left(\pi_{j}\right)^{2}-\frac{1}{12}\left\{\left(\mathcal{J}_{1}\right)^{2}+\left(\mathcal{J}_{2}\right)^{2}+\left(\mathcal{J}_{1} \mathcal{J}_{2}\right)\right\}+\frac{1}{24}\left(\phi_{j, x}\right)^{2}+i \widetilde{\psi}_{R}^{j} \psi_{R, x}^{j}-i \widetilde{\psi}_{L}^{j} \psi_{L, x}^{j} \\
& +i m_{\psi}^{j}\left(e^{-i \phi_{j}} \widetilde{\psi}_{R}^{j} \psi_{L}^{j}-e^{i \phi_{j}} \widetilde{\psi}_{L}^{j} \psi_{R}^{j}\right) .
\end{aligned}
$$

The new Lagrangian becomes

$$
\mathcal{L}^{\prime}=\pi_{1} \dot{\phi}_{1}+\pi_{2} \dot{\phi}_{2}+\dot{\psi}_{R}^{j} \pi_{R}^{j}+\dot{\psi}_{L}^{j} \pi_{L}^{j}-\mathcal{H}_{c}^{\prime} \text {. }
$$

We implement the constraints (3.8) by replacing in (3.10) the fields $\phi_{1}, \phi_{2}$ in terms of the space integral of the current components $J_{1}^{0}, J_{2}^{0}$. Then we get the Lagrangian

$$
\begin{aligned}
\mathcal{L}^{\prime \prime}= & \pi_{1} \partial_{t} \int^{x} J_{1}^{0}+\pi_{2} \partial_{t} \int^{x} J_{2}^{0}+\dot{\psi}_{R}^{j} \pi_{R}^{j}+\dot{\psi}_{L}^{j} \pi_{L}^{j}-i \widetilde{\psi}_{R}^{j} \psi_{R, x}^{j}+i \widetilde{\psi}_{L}^{j} \psi_{L, x}^{j}-i m_{\psi}^{j}\left(e^{-i \int^{x} J_{j}^{0}} \widetilde{\psi}_{R}^{j} \psi_{L}^{j}\right. \\
& \left.-e^{i \int^{x} J_{j}^{0}} \widetilde{\psi}_{L}^{j} \psi_{R}^{j}\right)-\frac{1}{12}\left(\left(J_{1}\right)^{2}+\left(J_{2}\right)^{2}+J_{1} \cdot J_{2}\right)+\pi_{1} J_{1}^{1}+\pi_{2} J_{2}^{1},
\end{aligned}
$$

where $J_{3}^{0} \equiv J_{1}^{0}+J_{2}^{0}$. Observe that the terms containig the $\pi_{a}$ 's in Eq. (3.11) cancel to each other if one uses the current conservation laws. Notice the appearances of various types of current-current interactions. The following Darboux transformation:

$$
\psi_{R}^{j} \rightarrow \exp \left(-\frac{i}{2} \int^{x} J_{j}^{0}\right) \psi_{R}^{j}, \quad \psi_{L}^{j} \rightarrow \exp \left(\frac{i}{2} \int^{x} J_{j}^{0}\right) \psi_{L}^{j}, \quad j=1,2,3
$$


is used to diagonalize the canonical one-form. Then, the kinetic terms will give additional currentcurrent interactions, $-\frac{1}{2}\left[J_{1} \cdot\left(j_{1}+j_{3}\right)+J_{2} \cdot\left(j_{2}+j_{3}\right)\right]$. We are, thus, after defining $k \equiv 1 / g$, and rescaling the fields $\psi^{j} \rightarrow 1 / \sqrt{k} \psi^{j}$, left with the Lagrangian

$$
\mathcal{L}[\psi, \bar{\psi}]=\sum_{j=1}^{3}\left\{i \bar{\psi}^{j} \gamma^{\mu} \partial_{\mu} \psi^{j}+m_{\psi}^{j} \bar{\psi}^{j} \psi^{j}\right\}-\sum_{\substack{k, l=1 \\ k \leqslant l}}^{3}\left[\bar{a}_{k l} j_{k} \cdot j_{l}\right],
$$

where $\bar{a}_{k l}=g a_{k l}$, with

$$
\begin{gathered}
a_{33}=\frac{1}{2}\left(\frac{\left(m^{3}\right)^{2}}{8}+m^{3}\right), \quad a_{12}=\frac{1}{12} m^{1} m^{2}, \quad a_{i i}=\frac{1}{2}\left(\frac{\left(m^{i}\right)^{2}}{6}+m^{i}\right), \\
a_{i 3}=\frac{1}{2}\left(\frac{m^{i} m^{3}}{4}+m^{i}+\frac{m^{3}}{2}\right), \quad i=1,2 .
\end{gathered}
$$

This defines the generalized massive Thirring model (GMT). The canonical pairs are $\left(-i \widetilde{\psi}_{R}^{j}, \psi_{R}^{j}\right)$ and $\left(-i \widetilde{\psi}_{L}^{j}, \psi_{L}^{j}\right)$.

\section{THE SYMPLECTIC FORMALISM AND THE ATM MODEL}

\section{A. The (constrained) symplectic formalism}

We give a brief overview of the basic notations of symplectic approach. ${ }^{24}$ The geometric structure is defined by the closed (pre)symplectic two-form

$$
f^{(0)}=\frac{1}{2} f_{i j}^{(0)}\left(\xi^{(0)}\right) \mathrm{d} \xi^{(0) i} \wedge \mathrm{d} \xi^{(0) j},
$$

where

$$
f_{i j}^{(0)}\left(\xi^{(0)}\right)=\frac{\partial}{\partial \xi^{(0) i}} \mathbf{a}_{j}^{(0)}\left(\xi^{(0)}\right)-\frac{\partial}{\partial \xi^{(0) j}} \mathbf{a}_{i}^{(0)}\left(\xi^{(0)}\right)
$$

with $\mathbf{a}^{(0)}\left(\xi^{(0)}\right)=\mathbf{a}_{j}^{(0)}\left(\xi^{(0)}\right) \mathrm{d} \xi^{(0) j}$ being the canonical one-form defined from the original first-order Lagrangian

$$
L^{(0)} \mathrm{d} t=\mathbf{a}^{(0)}\left(\xi^{(0)}\right)-V^{(0)}\left(\xi^{(0)}\right) \mathrm{d} t .
$$

The superscript (0) refers to the original Lagrangian, and is indicative of the iterative nature of the computations. The constraints are imposed through Lagrange multipliers which are velocities, and in such case one has to extend the configuration space. ${ }^{14,15}$ The corresponding Lagrangian gets modified and consequently the superscript also changes. The algorithm terminates once the symplectic matrix turns out to be nonsingular.

\section{B. The generalized massive Thirring model (GMT)}

Next, we will consider our model in the framework of the symplectic formalism. Let $\mathcal{L}^{\prime}$, Eq. (3.10), be the zeroth-iterated Lagrangian $\mathcal{L}^{(0)}$. Then the first iterated Lagrangian will be

$$
\mathcal{L}^{(1)}=\pi_{1} \dot{\phi}_{1}+\pi_{2} \dot{\phi}_{2}+\dot{\psi}_{R}^{j} \pi_{R}^{j}+\dot{\psi}_{L}^{j} \pi_{L}^{j}+\dot{\eta}^{1} \Omega_{1}+\dot{\eta}^{2} \Omega_{2}-\mathcal{V}^{(1)},
$$

where the once-iterated symplectic potential is defined by

$$
\mathcal{V}^{(1)}=\left.\mathcal{H}_{c}^{\prime}\right|_{\Omega_{1}=\Omega_{2}=0},
$$


and the stability conditions of the symplectic constraints, $\Omega_{1}$ and $\Omega_{2}$, under time evolution have been implemented by making $\lambda_{0}^{1} \rightarrow \dot{\eta}^{1}$ and $\lambda_{0}^{2} \rightarrow \dot{\eta}^{2}$. Consider the once-iterated set of symplectic variables in the following order

$$
\xi^{(\mathbf{1})}=\left(\eta^{1}, \eta^{2}, \phi_{1}, \phi_{2}, \psi_{R}^{1}, \psi_{L}^{1}, \psi_{R}^{2}, \psi_{L}^{2}, \psi_{R}^{3}, \psi_{L}^{3}, \pi_{1}, \pi_{2}, \pi_{R}^{1}, \pi_{L}^{1}, \pi_{R}^{2}, \pi_{L}^{2}, \pi_{R}^{3}, \pi_{L}^{3}\right),
$$

and the components of the canonical one-form

$$
\mathbf{a}^{(\mathbf{1})}=\left(\Omega_{1}, \Omega_{2}, \pi_{1}, \pi_{2},-\pi_{R}^{1},-\pi_{L}^{1},-\pi_{R}^{2},-\pi_{L}^{2},-\pi_{R}^{3},-\pi_{L}^{3}, 0,0,0,0,0,0,0,0\right) .
$$

These result in the singular symplectic two-form $18 \times 18$ matrix

$$
f_{A B}^{(1)}(x, y)=\left(\begin{array}{ll}
a_{11} & a_{12} \\
a_{21} & a_{22}
\end{array}\right) \delta(x-y),
$$

where the $9 \times 9$ martices are

$$
a_{11}=\left|\begin{array}{ccccccccc}
0 & 0 & \partial_{x} & 0 & i m^{1} \widetilde{\psi}_{R}^{1} & i m^{1} \widetilde{\psi}_{L}^{1} & 0 & 0 & \frac{i m^{3}}{2} \widetilde{\psi}_{R}^{3} \\
0 & 0 & 0 & \partial_{x} & 0 & 0 & i m^{2} \widetilde{\psi}_{R}^{2} & i m^{2} \widetilde{\psi}_{L}^{2} & \frac{i m^{3}}{2} \widetilde{\psi}_{R}^{3} \\
\partial_{x} & 0 & 0 & 0 & 0 & 0 & 0 & 0 & 0 \\
0 & \partial_{x} & 0 & 0 & 0 & 0 & 0 & 0 & 0 \\
i m^{1} \widetilde{\psi}_{R}^{1} & 0 & 0 & 0 & 0 & 0 & 0 & 0 & 0 \\
i m^{1} \widetilde{\psi}_{L}^{1} & 0 & 0 & 0 & 0 & 0 & 0 & 0 & 0 \\
0 & i m^{2} \widetilde{\psi}_{R}^{2} & 0 & 0 & 0 & 0 & 0 & 0 & 0 \\
0 & i m^{2} \widetilde{\psi}_{L}^{2} & 0 & 0 & 0 & 0 & 0 & 0 & 0 \\
\frac{i m^{3}}{2} \widetilde{\psi}_{R}^{3} & \frac{i m^{3}}{2} \widetilde{\psi}_{R}^{3} & 0 & 0 & 0 & 0 & 0 & 0 & 0 \\
\frac{i m^{3}}{2} \widetilde{\psi}_{L}^{3} & 0 & 0 & 0 & 0 & m^{2} \psi_{R}^{2} & m^{2} \psi_{L}^{2} & \frac{m^{3}}{2} \psi_{R}^{3} & \frac{m^{3}}{2} \psi_{L}^{3} \\
0 & -1 & 0 & 0 & 0 & 0 & 0 & 0 & 0 \\
\vdots & 0 & \ddots & \vdots & \vdots & \vdots & \vdots & \vdots & \vdots \\
0 & 0 & 0 & 0 & 0 & 0 & 0 & -1 & 0
\end{array}\right|,
$$




$$
a_{21}=\left(\begin{array}{ccccccccc}
\frac{i m^{3}}{2} \widetilde{\psi}_{L}^{3} & \frac{i m^{3}}{2} \widetilde{\psi}_{L}^{3} & 0 & 0 & 0 & 0 & 0 & 0 & 0 \\
0 & 0 & 1 & 0 & 0 & 0 & 0 & 0 & 0 \\
0 & 0 & 0 & 1 & 0 & 0 & 0 & 0 & 0 \\
m^{1} \psi_{R}^{1} & 0 & 0 & 0 & -1 & 0 & 0 & 0 & 0 \\
m^{1} \psi_{L}^{1} & 0 & 0 & 0 & 0 & -1 & 0 & 0 & 0 \\
0 & m^{2} \psi_{R}^{2} & 0 & 0 & 0 & 0 & -1 & 0 & 0 \\
0 & m^{2} \psi_{L}^{2} & 0 & 0 & 0 & 0 & 0 & -1 & 0 \\
\frac{m^{3}}{2} \psi_{R}^{3} & \frac{m^{3}}{2} \psi_{R}^{3} & 0 & 0 & 0 & 0 & 0 & 0 & -1 \\
\frac{m^{3}}{2} \psi_{L}^{3} & \frac{m^{3}}{2} \psi_{L}^{3} & 0 & 0 & 0 & 0 & 0 & 0 & 0
\end{array} \mid,\right.
$$

This matrix has the zero modes

$$
\begin{aligned}
\mathbf{v}^{(\mathbf{1}) T}(x)= & \left(\frac{-u}{m^{1}}, \frac{-v}{m^{2}}, 0,0, u \psi_{R}^{1}, u \psi_{L}^{1}, v \psi_{R}^{2}, v \psi_{L}^{2}, \frac{m^{3}}{2}\left(\frac{u}{m^{1}}+\frac{v}{m^{2}}\right) \psi_{R}^{3}, \frac{m^{3}}{2}\left(\frac{u}{m^{1}}+\frac{v}{m^{2}}\right) \psi_{L}^{3},-\frac{u^{\prime}}{m^{1}},\right. \\
& \left.-\frac{v^{\prime}}{m^{2}}, \text { іи } \widetilde{\psi}_{R}^{1}, \text { iи } \widetilde{\psi}_{L}^{1}, i v \widetilde{\psi}_{R}^{2}, i v \widetilde{\psi}_{L}^{2}, i \frac{m^{3}}{2}\left(\frac{u}{m^{1}}+\frac{v}{m^{2}}\right) \widetilde{\psi}_{R}^{3}, \frac{i m^{3}}{2}\left(\frac{u}{m^{1}}+\frac{v}{m^{2}}\right) \widetilde{\psi}_{L}^{3}\right),
\end{aligned}
$$

where $u$ and $v$ are arbitrary functions. The zero-mode condition gives

$$
\int \mathrm{d} x \mathbf{v}^{(\mathbf{1}) T}(x) \frac{\delta}{\delta \xi^{(1)}(x)} \int \mathrm{d} y \mathcal{V}^{(1)} \equiv 0 .
$$

Thus, the gradient of the symplectic potential happens to be orthogonal to the zero-mode $\mathbf{v}^{(1)}$. Since the equations of motion are automatically validated no symplectic constraints appear. This happens due to the presence of the symmetries of the action

$$
\delta \xi_{A}^{(1)}=\mathbf{v}_{\mathbf{A}}^{(1)}(x), \quad A=1,2, \ldots, 18 .
$$

So, in order to deform the symplectic matrix into an invertible one, we have to add some gauge fixing terms to the symplectic potential. One can choose any consistent set of gauge fixing conditions. ${ }^{15}$ In our case we have two symmetry generators associated with the parameters $u$ and $v$, so there must be two gauge conditions. Let us choose

$$
\Omega_{3} \equiv \phi_{1}=0, \quad \Omega_{4} \equiv \phi_{2}=0 .
$$

These conditions gauge away the fields $\phi_{1}$ and $\phi_{2}$, so only the remaining field variables will describe the dynamics of the system. Other gauge conditions, which eventually gauge away the spinor fields $\psi^{i}$, will be considered in Sec. IV C. 
Implementing the consistency conditions by means of Lagrange multipliers $\eta^{3}$ and $\eta^{4}$ we get the twice-iterated Lagrangian

$$
\mathcal{L}^{(2)}=\pi_{1} \dot{\phi}_{1}+\pi_{2} \dot{\phi}_{2}+\dot{\psi}_{R} \pi_{R}^{j}+\dot{\psi}_{L} \pi_{L}^{j}+\dot{\eta}^{1} \Omega_{1}+\dot{\eta}^{2} \Omega_{2}+\dot{\eta}^{3} \Omega_{3}+\dot{\eta}^{4} \Omega_{4}-\mathcal{V}^{(2)},
$$

where

$$
\mathcal{V}^{(2)}=\left.\mathcal{V}^{(1)}\right|_{\Omega_{3}=\Omega_{4}=0}
$$

Assuming now that the new set of symplectic variables is given in the following order,

$$
\xi^{(2)}=\left(\eta^{1}, \eta^{2}, \eta^{3}, \eta^{4}, \phi_{1}, \phi_{2}, \psi_{R}^{1}, \psi_{L}^{1}, \psi_{R}^{2}, \psi_{L}^{2}, \psi_{R}^{3}, \psi_{L}^{3}, \pi_{1}, \pi_{2}, \pi_{R}^{1}, \pi_{L}^{1}, \pi_{R}^{2}, \pi_{L}^{2}, \pi_{R}^{3}, \pi_{L}^{3}\right)
$$

and the nonvanishing components of the canonical one-form

$$
\mathbf{a}^{(2)}=\left(\Omega_{1}, \Omega_{2}, \Omega_{3}, \Omega_{4}, \pi_{1}, \pi_{2},-\pi_{R}^{1},-\pi_{L}^{1},-\pi_{R}^{2},-\pi_{L}^{2},-\pi_{R}^{3},-\pi_{L}^{3}, 0,0,0,0,0,0,0,0\right),
$$

one obtains the singular twice-iterated symplectic $20 \times 20$ matrix

$$
f_{A B}^{(2)}(x, y)=\left(\begin{array}{ll}
a_{11} & a_{12} \\
a_{21} & a_{22}
\end{array}\right) \delta(x-y)
$$

where the $10 \times 10$ matrices are

$$
a_{11}=\left(\begin{array}{cccccccccc}
0 & 0 & 0 & 0 & \partial_{x} & 0 & i m^{1} \widetilde{\psi}_{R}^{1} & i m^{1} \widetilde{\psi}_{L}^{1} & 0 & 0 \\
0 & 0 & 0 & 0 & 0 & \partial_{x} & 0 & 0 & i m^{2} \widetilde{\psi}_{R}^{2} & i m^{2} \widetilde{\psi}_{L}^{2} \\
0 & 0 & 0 & 0 & -1 & 0 & 0 & 0 & 0 & 0 \\
0 & 0 & 0 & 0 & 0 & -1 & 0 & 0 & 0 & 0 \\
\partial_{x} & 0 & 1 & 0 & 0 & 0 & 0 & 0 & 0 & 0 \\
0 & \partial_{x} & 0 & 1 & 0 & 0 & 0 & 0 & 0 & 0 \\
i m^{1} \widetilde{\psi}_{R}^{1} & 0 & 0 & 0 & 0 & 0 & 0 & 0 & 0 & 0 \\
i m^{1} \widetilde{\psi}_{L}^{1} & 0 & 0 & 0 & 0 & 0 & 0 & 0 & 0 & 0 \\
0 & i m^{2} \widetilde{\psi}_{R}^{2} & 0 & 0 & 0 & 0 & 0 & 0 & 0 & 0 \\
0 & i m^{2} \widetilde{\psi}_{L}^{2} & 0 & 0 & 0 & 0 & 0 & 0 & 0 & 0
\end{array}\right),
$$




$$
a_{12}=\left(\begin{array}{cccccccccc}
\frac{i m^{3}}{2} \widetilde{\psi}_{R}^{3} & \frac{i m^{3}}{2} \widetilde{\psi}_{L}^{3} & 0 & 0 & m^{1} \psi_{R}^{1} & m^{1} \psi_{L}^{1} & 0 & 0 & \frac{m^{3}}{2} \psi_{R}^{3} & \frac{m^{3}}{2} \psi_{L}^{3} \\
\frac{i m^{3}}{2} \widetilde{\psi}_{R}^{3} & \frac{i m^{3}}{2} \widetilde{\psi}_{L}^{3} & 0 & 0 & 0 & 0 & m^{2} \psi_{R}^{2} & m^{2} \psi_{L}^{2} & \frac{m^{3}}{2} \psi_{R}^{3} & \frac{m^{3}}{2} \psi_{L}^{3} \\
0 & 0 & 0 & 0 & 0 & 0 & 0 & 0 & 0 & 0 \\
0 & 0 & 0 & 0 & 0 & 0 & 0 & 0 & 0 & 0 \\
0 & 0 & -1 & 0 & 0 & 0 & 0 & 0 & 0 & 0 \\
0 & 0 & 0 & -1 & 0 & 0 & 0 & 0 & 0 & 0 \\
0 & 0 & 0 & 0 & -1 & 0 & 0 & 0 & 0 & 0 \\
0 & 0 & 0 & 0 & 0 & -1 & 0 & 0 & 0 & 0 \\
0 & 0 & 0 & 0 & 0 & 0 & -1 & 0 & 0 & 0 \\
0 & 0 & 0 & 0 & 0 & 0 & 0 & -1 & 0 & 0
\end{array}\right),
$$

$$
a_{21}=\left(\begin{array}{cccccccccc}
\frac{i m^{3}}{2} \widetilde{\psi}_{R}^{3} & \frac{i m^{3}}{2} \widetilde{\psi}_{R}^{3} & 0 & 0 & 0 & 0 & 0 & 0 & 0 & 0 \\
\frac{i m^{3}}{2} \widetilde{\psi}_{L}^{3} & \frac{i m^{3}}{2} \widetilde{\psi}_{L}^{3} & 0 & 0 & 0 & 0 & 0 & 0 & 0 & 0 \\
0 & 0 & 0 & 0 & 1 & 0 & 0 & 0 & 0 & 0 \\
0 & 0 & 0 & 0 & 0 & 1 & 0 & 0 & 0 & 0 \\
m^{1} \psi_{R}^{1} & 0 & 0 & 0 & 0 & 0 & -1 & 0 & 0 & 0 \\
m^{1} \psi_{L}^{1} & 0 & 0 & 0 & 0 & 0 & 0 & -1 & 0 & 0 \\
0 & m^{2} \psi_{R}^{2} & 0 & 0 & 0 & 0 & 0 & 0 & -1 & 0 \\
0 & m^{2} \psi_{L}^{2} & 0 & 0 & 0 & 0 & 0 & 0 & 0 & -1 \\
\frac{m^{3}}{2} \psi_{R}^{3} & \frac{m^{3}}{2} \psi_{R}^{3} & 0 & 0 & 0 & 0 & 0 & 0 & 0 & 0 \\
\frac{m^{3}}{2} \psi_{L}^{3} & \frac{m^{3}}{2} \psi_{L}^{3} & 0 & 0 & 0 & 0 & 0 & 0 & 0 & 0
\end{array}\right),
$$

$$
a_{22}=\left(\begin{array}{ccccccc}
0 & 0 & 0 & \cdots & 0 & -1 & 0 \\
0 & 0 & 0 & \cdots & 0 & 0 & -1 \\
0 & 0 & 0 & \cdots & 0 & 0 & 0 \\
& \cdots \cdots \cdots \cdots \cdots \cdots \cdots \cdots \cdots \cdots \cdots & \cdots \\
0 & 0 & 0 & \cdots & 0 & 0 & 0 \\
-1 & 0 & 0 & \cdots & 0 & 0 & 0 \\
0 & -1 & 0 & \cdots & 0 & 0 & 0
\end{array}\right) .
$$

The zero-modes are 


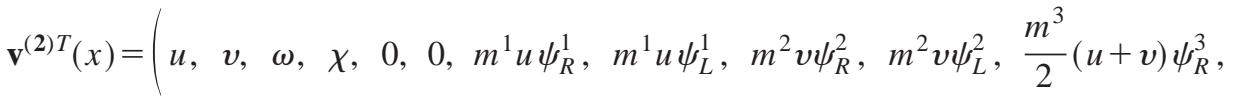

$$
\begin{aligned}
& \frac{m^{3}}{2}(u+v) \psi_{L}^{3}, \quad u^{\prime}+\omega, \quad v^{\prime}+\chi, i m^{1} \widetilde{\psi}_{R}^{1} u, i m^{1} \widetilde{\psi}_{L}^{1} u, i m^{2} \widetilde{\psi}_{R}^{2} v, i m^{2} \widetilde{\psi}_{L}^{2} v, \\
& \left.\frac{i m^{3}}{2} \widetilde{\psi}_{R}^{3}(u+v), i \frac{m^{3}}{2} \widetilde{\psi}_{L}^{3}(u+v)\right) \text {. }
\end{aligned}
$$

The zero-mode condition gives no constraints, implying the symmetries of the action

$$
\delta \xi_{\mathbf{A}}^{(\mathbf{2})}=\mathbf{v}_{\mathbf{A}}^{(\mathbf{2})}(x), \quad A=1,2, \ldots, 20 .
$$

Now, let us choose the gauge conditions

$$
\Omega_{5} \equiv \pi_{1} J_{1}^{1}+\frac{1}{2} J_{1} \cdot\left(j_{1}+j_{3}\right)=0, \quad \Omega_{6} \equiv \pi_{2} J_{2}^{1}+\frac{1}{2} J_{2} \cdot\left(j_{2}+j_{3}\right)=0,
$$

and impose the consistency conditions with the Lagrange multipliers $\eta^{5}, \eta^{6}$, then

$$
\mathcal{L}^{(3)}=\pi_{1} \dot{\phi}_{1}+\pi_{2} \dot{\phi}_{2}+\dot{\psi}_{R} \pi_{R}^{j}+\dot{\psi}_{L} \pi_{L}^{j}+\dot{\eta}^{1} \Omega_{1}+\dot{\eta}^{2} \Omega_{2}+\dot{\eta}^{3} \Omega_{3}+\dot{\eta}^{4} \Omega_{4}+\dot{\eta}^{5} \Omega_{5}+\dot{\eta}^{6} \Omega_{6}-\mathcal{V}^{(3)}
$$

where

$$
\mathcal{V}^{(3)}=\left.\mathcal{V}^{(2)}\right|_{\Omega_{5}=\Omega_{6}=0},
$$

or explicitly

$\mathcal{V}^{(3)}=\frac{1}{12}\left(\left(J_{1}\right)^{2}+\left(J_{2}\right)^{2}+J_{1} \cdot J_{2}\right)+\frac{1}{2}\left[J_{1} \cdot\left(j_{1}+j_{3}\right)+J_{2} \cdot\left(j_{2}+j_{3}\right)\right]+i \widetilde{\psi}_{R}^{j} \psi_{R, x}^{j}-i \widetilde{\psi}_{L}^{j} \psi_{L, x}^{j}+i m_{\psi}^{j} \bar{\psi}^{j} \psi^{j}$.

The symplectic two-form for this Lagrangian is a nonsingular matrix, then our algorithm has come to an end. Collecting the canonical part and the symplectic potential $\mathcal{V}^{(3)}$ one has

$$
\mathcal{L}[\psi, \bar{\psi}]=\sum_{j=1}^{3}\left\{i \bar{\psi}^{j} \gamma^{\mu} \partial_{\mu} \psi^{j}+m_{\psi}^{j} \bar{\psi}^{j} \psi^{j}\right\}-\sum_{\substack{k, l=1 \\ k \leqslant l}}^{3}\left[\bar{a}_{k l} j_{k} \cdot j_{l}\right]+\sum_{l=1}^{3} m^{l} \nu_{l} j_{l}^{0},
$$

where $\nu_{3} \equiv\left(\nu_{1}+\nu_{2}\right) / 2$. We have made the same choice, $k=1 / g$, and the field rescalings $\psi^{j}$ $\rightarrow 1 / \sqrt{k} \psi^{j}$ as in the last section. This is the same GMT Lagrangian as (3.13). As a bonus, we get the chemical potentials $\mu_{l} \equiv m^{l} \nu_{l}\left(\dot{\eta}^{1,2} \rightarrow \nu_{1,2}\right)$ times the charge densities. These terms are related to the charges $Q_{F}^{l}=1 / 2 \pi \int_{-\infty}^{+\infty} \mathrm{d} x j_{l}^{0}(t, x)$, and their presence is a consequence of the symplectic method. ${ }^{11}$

\section{The generalized sine-Gordon model (GSG)}

One can choose other gauge fixings, instead of (4.12), to construct the twice-iterated Lagrangian. Let us make the choice

$$
\Omega_{3} \equiv J_{1}^{0}=0, \quad \Omega_{4} \equiv J_{2}^{0}=0,
$$

which satisfies the nongauge invariance condition as can be verified by computing the brackets $\left\{\Omega_{a}, J_{b}^{0}\right\}=0 ; a, b=1,2$. The twice-iterated Lagrangian is obtained by bringing back these constraints into the canonical part of $\mathcal{L}^{(1)}$, then

$$
\mathcal{L}^{(2)}=\pi_{1} \dot{\phi}_{1}+\pi_{2} \dot{\phi}_{2}+\dot{\psi}_{R} \pi_{R}^{j}+\dot{\psi}_{L} \pi_{L}^{j}+\dot{\eta}^{1} \Omega_{1}+\dot{\eta}^{2} \Omega_{2}+\dot{\eta}^{3} \Omega_{3}+\dot{\eta}^{4} \Omega_{4}-\mathcal{V}^{(2)},
$$


where the twice-iterated symplectic potential becomes

$$
\mathcal{V}^{(2)}=\left.\mathcal{V}^{(1)}\right|_{\Omega_{3}=\Omega_{4}=0} .
$$

Considering the set of symplectic variables in the following order:

$$
\xi_{A}^{(2)}=\left(\eta^{1}, \eta^{2}, \eta^{3}, \eta^{4}, \phi_{1}, \phi_{2}, \psi_{R}^{1}, \psi_{L}^{1}, \psi_{R}^{2}, \psi_{L}^{2}, \psi_{R}^{3}, \psi_{L}^{3}, \pi_{1}, \pi_{2}, \pi_{R}^{1}, \pi_{L}^{1}, \pi_{R}^{2}, \pi_{L}^{2}, \pi_{R}^{3}, \pi_{L}^{3}\right)
$$

and the components of the canonical one-form

$$
a_{A}^{(2)}=\left(\Omega_{1}, \Omega_{2}, \Omega_{3}, \Omega_{4}, \pi_{1}, \pi_{2},-\pi_{R}^{1},-\pi_{L}^{1},-\pi_{R}^{2},-\pi_{L}^{2},-\pi_{R}^{3},-\pi_{L}^{3}, 0,0,0,0,0,0,0,0\right),
$$

the (degenerated) $20 \times 20$ symplectic matrix is found to be

$$
f_{A B}^{(2)}(x, y)=\left(\begin{array}{ll}
a_{11} & a_{12} \\
a_{21} & a_{22}
\end{array}\right) \delta(x-y),
$$

where

$$
a_{11}=\left(\begin{array}{cccccccccc}
0 & 0 & 0 & 0 & \partial_{x} & 0 & i m^{1} \widetilde{\psi}_{R}^{1} & i m^{1} \widetilde{\psi}_{L}^{1} & 0 & 0 \\
0 & 0 & 0 & 0 & 0 & \partial_{x} & 0 & 0 & i m^{2} \widetilde{\psi}_{R}^{2} & i m^{2} \widetilde{\psi}_{L}^{2} \\
0 & 0 & 0 & 0 & 0 & 0 & i m^{1} \widetilde{\psi}_{R}^{1} & i m^{1} \widetilde{\psi}_{L}^{1} & 0 & 0 \\
0 & 0 & 0 & 0 & 0 & 0 & 0 & 0 & i m^{2} \widetilde{\psi}_{R}^{2} & i m^{2} \widetilde{\psi}_{L}^{2} \\
\partial_{x} & 0 & 0 & 0 & 0 & 0 & 0 & 0 & 0 & 0 \\
0 & \partial_{x} & 0 & 0 & 0 & 0 & 0 & 0 & 0 & 0 \\
i m^{1} \widetilde{\psi}_{R}^{1} & 0 & i m^{1} \widetilde{\psi}_{R}^{1} & 0 & 0 & 0 & 0 & 0 & 0 & 0 \\
i m^{1} \widetilde{\psi}_{L}^{1} & 0 & i m^{1} \widetilde{\psi}_{L}^{1} & 0 & 0 & 0 & 0 & 0 & 0 & 0 \\
0 & i m^{2} \widetilde{\psi}_{R}^{2} & 0 & i m^{2} \widetilde{\psi}_{R}^{2} & 0 & 0 & 0 & 0 & 0 & 0 \\
0 & i m^{2} \widetilde{\psi}_{L}^{2} & 0 & i m^{2} \widetilde{\psi}_{L}^{2} & 0 & 0 & 0 & 0 & 0 & 0 \\
\frac{i m^{3}}{2} \widetilde{\psi}_{R}^{3} & \frac{i m^{3}}{2} \widetilde{\psi}_{L}^{3} & 0 & 0 & m^{1} \psi_{R}^{1} & m^{1} \psi_{L}^{1} & 0 & 0 & \frac{m^{3}}{2} \psi_{R}^{3} & \frac{m^{3}}{2} \psi_{L}^{3} \\
\frac{i m^{3}}{2} \widetilde{\psi}_{R}^{3} & \frac{i m^{3}}{2} \widetilde{\psi}_{L}^{3} & 0 & 0 & 0 & 0 & m^{2} \psi_{R}^{2} & m^{2} \psi_{L}^{2} & \frac{m^{3}}{2} \psi_{R}^{3} & \frac{m^{3}}{2} \psi_{L}^{3} \\
\frac{i m^{3}}{2} \widetilde{\psi}_{R}^{3} & \frac{i m^{3}}{2} \widetilde{\psi}_{L}^{3} & 0 & 0 & m^{1} \psi_{R}^{1} & m^{1} \psi_{L}^{1} & 0 & 0 & \frac{m^{3}}{2} \psi_{R}^{3} & \frac{m^{3}}{2} \psi_{L}^{3} \\
\frac{i m^{3}}{2} \widetilde{\psi}_{R}^{3} & \frac{i m^{3}}{2} \widetilde{\psi}_{L}^{3} & 0 & 0 & 0 & 0 & m^{2} \psi_{R}^{2} & m^{2} \psi_{L}^{2} & \frac{m^{3}}{2} \psi_{R}^{3} & \frac{m^{3}}{2} \psi_{L}^{3} \\
0 & 0 & -1 & 0 & 0 & 0 & 0 & 0 & 0 & 0 \\
0 & 0 & 0 & -1 & 0 & 0 & 0 & 0 & 0 & 0 \\
0 & 0 & 0 & 0 & -1 & 0 & 0 & 0 & 0 & 0 \\
0 & 0 & 0 & 0 & 0 & -1 & 0 & 0 & 0 & 0 \\
0 & 0 & 0 & 0 & 0 & 0 & 0 & -1 & 0 & 0
\end{array}\right),
$$




$$
\begin{aligned}
& a_{21}=\left(\begin{array}{cccccccccc}
\frac{i m^{3}}{2} \widetilde{\psi}_{R}^{3} & \frac{i m^{3}}{2} \widetilde{\psi}_{R}^{3} & \frac{i m^{3}}{2} \widetilde{\psi}_{R}^{3} & \frac{i m^{3}}{2} \widetilde{\psi}_{R}^{3} & 0 & 0 & 0 & 0 & 0 & 0 \\
\frac{i m^{3}}{2} \widetilde{\psi}_{L}^{3} & \frac{i m^{3}}{2} \widetilde{\psi}_{L}^{3} & \frac{i m^{3}}{2} \widetilde{\psi}_{L}^{3} & \frac{i m^{3}}{2} \widetilde{\psi}_{L}^{3} & 0 & 0 & 0 & 0 & 0 & 0 \\
0 & 0 & 0 & 0 & 1 & 0 & 0 & 0 & 0 & 0 \\
0 & 0 & 0 & 0 & 0 & 1 & 0 & 0 & 0 & 0 \\
m^{1} \psi_{R}^{1} & 0 & m^{1} \psi_{R}^{1} & 0 & 0 & 0 & -1 & 0 & 0 & 0 \\
m^{1} \psi_{L}^{1} & 0 & m^{1} \psi_{L}^{1} & 0 & 0 & 0 & 0 & -1 & 0 & 0 \\
0 & m^{2} \psi_{R}^{2} & 0 & m^{2} \psi_{R}^{2} & 0 & 0 & 0 & 0 & -1 & 0 \\
0 & m^{2} \psi_{L}^{2} & 0 & m^{2} \psi_{L}^{2} & 0 & 0 & 0 & 0 & 0 & -1 \\
\frac{m^{3}}{2} \psi_{R}^{3} & \frac{m^{3}}{2} \psi_{R}^{3} & \frac{m^{3}}{2} \psi_{R}^{3} & \frac{m^{3}}{2} \psi_{R}^{3} & 0 & 0 & 0 & 0 & 0 & 0 \\
\frac{m^{3}}{2} \psi_{L}^{3} & \frac{m^{3}}{2} \psi_{L}^{3} & \frac{m^{3}}{2} \psi_{L}^{3} & \frac{m^{3}}{2} \psi_{L}^{3} & 0 & 0 & 0 & 0 & 0 & 0
\end{array}\right), \\
& a_{22}=\left(\begin{array}{ccccccc}
0 & 0 & 0 & \cdots & 0 & -1 & 0 \\
0 & 0 & 0 & \cdots & 0 & 0 & -1 \\
0 & 0 & 0 & \cdots & 0 & 0 & 0 \\
0 & 0 & 0 & \cdots & 0 & 0 & 0 \\
-1 & 0 & 0 & \cdots & 0 & 0 & 0 \\
0 & -1 & 0 & \cdots & 0 & 0 & 0
\end{array}\right) .
\end{aligned}
$$

Its zero modes are

$$
\begin{aligned}
\mathbf{v}^{(2) T}(x)= & \left(u, \quad v, \omega, \chi, 0,0, m^{1}(u+\omega) \psi_{R}^{1}, m^{1}(u+\omega) \psi_{L}^{1}, m^{2}(v+\chi) \psi_{R}^{2}, m^{2}(v+\chi) \psi_{L}^{2},\right. \\
& \frac{m^{3}}{2}(u+v+\omega+\chi) \psi_{R}^{3}, \frac{m^{3}}{2}(u+v+\omega+\chi) \psi_{L}^{3}, \quad u^{\prime}, v^{\prime}, i m^{1} \widetilde{\psi}_{R}^{1}(u+\omega), \\
& i m^{1} \widetilde{\psi}_{L}^{1}(u+\omega), i m^{2} \widetilde{\psi}_{R}^{2}(v+\chi), i m^{2} \widetilde{\psi}_{L}^{2}(v+\chi), \frac{i m^{3}}{2} \widetilde{\psi}_{R}^{3}(u+v+\omega+\chi) \\
& \left.\frac{i m^{3}}{2} \widetilde{\psi}_{L}^{3}(u+v+\omega+\chi)\right)
\end{aligned}
$$

where $u, v, \omega$, and $\chi$ are arbitrary functions. The zero-mode condition becomes

$$
\int \mathrm{d} x \mathbf{v}^{(2) T}(x) \frac{\delta}{\delta \xi^{(2)}} \int \mathrm{d} y^{\prime} \mathcal{V}^{(2)}=\int \mathrm{d} x J_{a}^{1} \partial_{x} f_{a} \equiv 0, \quad f_{a} \equiv(\omega, \chi), \quad a=1,2
$$

Since the functions $f_{a}$ are arbitrary we end up with the following constraints:

$$
\Omega_{5} \equiv J_{1}^{1}=0, \quad \Omega_{6} \equiv J_{2}^{1}=0 .
$$

Notice that by solving the constraints, $\Omega_{3}=\Omega_{4}=\Omega_{5}=\Omega_{6}=0$, Eqs. (4.24) and (4.31), we may obtain 


$$
\widetilde{\psi}_{R}^{j}=\psi_{R}^{j}, \quad \widetilde{\psi}_{L}^{j}=\psi_{L}^{j}
$$

So, at this stage, we have Majorana spinors, the scalars $\phi_{1}$ and $\phi_{2}$, and the auxiliary fields. Next, introduce a third set of Lagrange multipliers into $\mathcal{L}^{(2)}$, then

$$
\mathcal{L}^{(3)}=\pi_{1} \dot{\phi}_{1}+\pi_{2} \dot{\phi}_{2}+\dot{\psi}_{R} \pi_{R}^{j}+\dot{\psi}_{L} \pi_{L}^{j}+\dot{\eta}^{1} \Omega_{1}+\dot{\eta}^{2} \Omega_{2}+\dot{\eta}^{3} \Omega_{3}+\dot{\eta}^{4} \Omega_{4}+\dot{\eta}^{5} \Omega_{5}+\dot{\eta}^{6} \Omega_{6}-\mathcal{V}^{(3)},
$$

where

$$
\mathcal{V}^{(3)}=\left.\mathcal{V}^{(2)}\right|_{\Omega_{5}=\Omega_{6}=0}
$$

or

$$
\mathcal{V}^{(3)}=\frac{1}{24} \phi_{j, x}^{2}+i \psi_{R}^{j} \psi_{R, x}^{j}-i \psi_{L}^{j} \psi_{L, x}^{j}+i m_{\psi}^{j} \psi_{R}^{j} \psi_{L}^{j}\left(e^{-i \phi_{j}}+e^{i \phi_{j}}\right)
$$

The new set of symplectic variables is assumed to be ordered as

$$
\xi_{A}^{(3)}=\left(\eta^{1}, \eta^{2}, \eta^{3}, \eta^{4}, \eta^{5}, \eta^{6}, \phi_{1}, \phi_{2}, \psi_{R}^{1}, \psi_{L}^{1}, \psi_{R}^{2}, \psi_{L}^{2}, \psi_{R}^{3}, \psi_{L}^{3}, \pi_{1}, \pi_{2}, \pi_{R}^{1}, \pi_{L}^{1}, \pi_{R}^{2}, \pi_{L}^{2}, \pi_{R}^{3}, \pi_{L}^{3}\right)
$$

The components of the canonical one-form are

$$
a_{A}^{(3)}=\left(\Omega_{1}, \Omega_{2}, \Omega_{3}, \Omega_{4}, \Omega_{5}, \Omega_{6}, \pi_{1}, \pi_{2},-\pi_{R}^{1},-\pi_{L}^{1},-\pi_{R}^{2},-\pi_{L}^{2},-\pi_{R}^{3},-\pi_{L}^{3}, 0,0,0,0,0,0,0,0\right) .
$$

After some algebraic manipulations we get the third-iterated $22 \times 22$ symplectic two-form

$$
f_{A B}^{(3)}(x, y)=\left(\begin{array}{ll}
a_{11} & a_{12} \\
a_{21} & a_{22}
\end{array}\right) \delta(x-y),
$$

where

$$
a_{11}=\left(\begin{array}{ccccccccccc}
0 & 0 & 0 & 0 & 0 & 0 & \partial_{x} & 0 & i m^{1} \widetilde{\psi}_{R}^{1} & i m^{1} \widetilde{\psi}_{L}^{1} & 0 \\
0 & 0 & 0 & 0 & 0 & 0 & 0 & \partial_{x} & 0 & 0 & i m^{2} \widetilde{\psi}_{R}^{2} \\
0 & 0 & 0 & 0 & 0 & 0 & 0 & 0 & i m^{1} \widetilde{\psi}_{R}^{1} & i m^{1} \widetilde{\psi}_{L}^{1} & 0 \\
0 & 0 & 0 & 0 & 0 & 0 & 0 & 0 & 0 & 0 & i m^{2} \widetilde{\psi}_{R}^{2} \\
0 & 0 & 0 & 0 & 0 & 0 & 0 & 0 & -i m^{1} \widetilde{\psi}_{R}^{1} & i m^{1} \widetilde{\psi}_{L}^{1} & 0 \\
0 & 0 & 0 & 0 & 0 & 0 & 0 & 0 & 0 & 0 & -i m^{2} \widetilde{\psi}_{R}^{2} \\
\partial_{x} & 0 & 0 & 0 & 0 & 0 & 0 & 0 & 0 & 0 & 0 \\
0 & \partial_{x} & 0 & 0 & 0 & 0 & 0 & 0 & 0 & 0 & 0 \\
i m^{1} \widetilde{\psi}_{R}^{1} & 0 & i m^{1} \widetilde{\psi}_{R}^{1} & 0 & -i m^{1} \widetilde{\psi}_{R}^{1} & 0 & 0 & 0 & 0 & 0 & 0 \\
i m^{1} \widetilde{\psi}_{L}^{1} & 0 & i m^{1} \widetilde{\psi}_{L}^{1} & 0 & i m^{1} \widetilde{\psi}_{L}^{1} & 0 & 0 & 0 & 0 & 0 & 0 \\
0 & i m^{2} \widetilde{\psi}_{R}^{2} & 0 & i m^{2} \widetilde{\psi}_{R}^{2} & 0 & -i m^{2} \widetilde{\psi}_{R}^{2} & 0 & 0 & 0 & 0 & 0
\end{array}\right)
$$




$$
\begin{aligned}
& a_{12}=\left(\begin{array}{ccccccccccc}
0 & \frac{i m^{3}}{2} \widetilde{\psi}_{R}^{3} & \frac{i m^{3}}{2} \widetilde{\psi}_{L}^{3} & 0 & 0 & m^{1} \psi_{R}^{1} & m^{1} \psi_{L}^{1} & 0 & 0 & \frac{m^{3}}{2} \psi_{R}^{3} & \frac{m^{3}}{2} \psi_{L}^{3} \\
i m^{2} \widetilde{\psi}_{L}^{2} & \frac{i m^{3}}{2} \widetilde{\psi}_{R}^{3} & \frac{i m^{3}}{2} \widetilde{\psi}_{L}^{3} & 0 & 0 & 0 & 0 & m^{2} \psi_{R}^{2} & m^{2} \psi_{L}^{2} & \frac{m^{3}}{2} \psi_{R}^{3} & \frac{m^{3}}{2} \psi_{L}^{3} \\
0 & \frac{i m^{3}}{2} \widetilde{\psi}_{R}^{3} & \frac{i m^{3}}{2} \widetilde{\psi}_{L}^{3} & 0 & 0 & m^{1} \psi_{R}^{1} & m^{1} \psi_{L}^{1} & 0 & 0 & \frac{m^{3}}{2} \psi_{R}^{3} & \frac{m^{3}}{2} \psi_{L}^{3} \\
i m^{2} \widetilde{\psi}_{L}^{2} & \frac{i m^{3}}{2} \widetilde{\psi}_{R}^{3} & \frac{i m^{3}}{2} \widetilde{\psi}_{L}^{3} & 0 & 0 & 0 & 0 & m^{2} \psi_{R}^{2} & m^{2} \psi_{L}^{2} & \frac{m^{3}}{2} \psi_{R}^{3} & \frac{m^{3}}{2} \psi_{L}^{3} \\
0 & -\frac{i m^{3}}{2} \widetilde{\psi}_{R}^{3} & \frac{i m^{3}}{2} \widetilde{\psi}_{L}^{3} & 0 & 0 & -m^{1} \psi_{R}^{1} & m^{1} \psi_{L}^{1} & 0 & 0 & -\frac{m^{2}}{2} \psi_{R}^{3} & \frac{m^{3}}{2} \psi_{L}^{3} \\
i m^{2} \widetilde{\psi}_{L}^{2} & -\frac{i m^{3}}{2} \widetilde{\psi}_{R}^{3} & \frac{i m^{3}}{2} \widetilde{\psi}_{L}^{3} & 0 & 0 & 0 & 0 & -m^{2} \psi_{R}^{2} & m^{2} \psi_{L}^{2} & -\frac{m^{3}}{2} \psi_{R}^{3} & \frac{m^{3}}{2} \psi_{L}^{3} \\
0 & 0 & 0 & -1 & 0 & 0 & 0 & 0 & 0 & 0 & 0 \\
0 & 0 & 0 & 0 & -1 & 0 & 0 & 0 & 0 & 0 & 0 \\
0 & 0 & 0 & 0 & 0 & -1 & 0 & 0 & 0 & 0 & 0 \\
0 & 0 & 0 & 0 & 0 & 0 & -1 & 0 & 0 & 0 & 0 \\
0 & 0 & 0 & 0 & 0 & 0 & -1 & 0 & 0 & 0
\end{array} \mid,\right. \\
& a_{21}=\left(\begin{array}{ccccccccccc}
0 & i m^{2} \widetilde{\psi}_{L}^{2} & 0 & i m^{2} \widetilde{\psi}_{L}^{2} & 0 & i m^{2} \widetilde{\psi}_{L}^{2} & 0 & 0 & 0 & 0 & 0 \\
\frac{i m^{3}}{2} \widetilde{\psi}_{R}^{3} & \frac{i m^{3}}{2} \widetilde{\psi}_{R}^{3} & \frac{i m^{3}}{2} \widetilde{\psi}_{R}^{3} & \frac{i m^{3}}{2} \widetilde{\psi}_{R}^{3} & -\frac{i m^{3}}{2} \widetilde{\psi}_{R}^{3} & -\frac{i m^{3}}{2} \widetilde{\psi}_{R}^{3} & 0 & 0 & 0 & 0 & 0 \\
\frac{i m^{3}}{2} \widetilde{\psi}_{L}^{3} & \frac{i m^{3}}{2} \widetilde{\psi}_{L}^{3} & \frac{i m^{3}}{2} \widetilde{\psi}_{L}^{3} & \frac{i m^{3}}{2} \widetilde{\psi}_{L}^{3} & \frac{i m^{3}}{2} \widetilde{\psi}_{L}^{3} & \frac{i m^{3}}{2} \widetilde{\psi}_{L}^{3} & 0 & 0 & 0 & 0 & 0 \\
0 & 0 & 0 & 0 & 0 & 0 & 1 & 0 & 0 & 0 & 0 \\
0 & 0 & 0 & 0 & 0 & 0 & 0 & 1 & 0 & 0 & 0 \\
m^{1} \psi_{R}^{1} & 0 & m^{1} \psi_{R}^{1} & 0 & -m^{1} \psi_{R}^{1} & 0 & 0 & 0 & -1 & 0 & 0 \\
m^{1} \psi_{L}^{1} & 0 & m^{1} \psi_{L}^{1} & 0 & m^{1} \psi_{L}^{1} & 0 & 0 & 0 & 0 & -1 & 0 \\
0 & m^{2} \psi_{R}^{2} & 0 & m^{2} \psi_{R}^{2} & 0 & -m^{2} \psi_{R}^{2} & 0 & 0 & 0 & 0 & -1 \\
0 & m^{2} \psi_{L}^{2} & 0 & m^{2} \psi_{L}^{2} & 0 & m^{2} \psi_{L}^{2} & 0 & 0 & 0 & 0 & 0 \\
\frac{m^{3}}{2} \psi_{R}^{3} & \frac{m^{3}}{2} \psi_{R}^{3} & \frac{m^{3}}{2} \psi_{R}^{3} & \frac{m^{3}}{2} \psi_{R}^{3} & -\frac{m^{3}}{2} \psi_{R}^{3} & -\frac{m^{3}}{2} \psi_{R}^{3} & 0 & 0 & 0 & 0 & 0 \\
\frac{m^{3}}{2} \psi_{L}^{3} & \frac{m^{3}}{2} \psi_{L}^{3} & \frac{m^{3}}{2} \psi_{L}^{3} & \frac{m^{3}}{2} \psi_{L}^{3} & \frac{m^{3}}{2} \psi_{L}^{3} & \frac{m^{3}}{2} \psi_{L}^{3} & 0 & 0 & 0 & 0 & 0
\end{array} \mid\right. \\
& a_{22}=\left(\begin{array}{ccccccccc}
0 & 0 & 0 & 0 & \cdots & 0 & -1 & 0 & 0 \\
0 & 0 & 0 & 0 & \cdots & 0 & 0 & -1 & 0 \\
0 & 0 & 0 & 0 & \cdots & 0 & 0 & 0 & -1 \\
0 & 0 & 0 & 0 & \cdots & 0 & 0 & 0 & 0 \\
0 & 0 & 0 & 0 & \cdots & 0 & 0 & 0 & 0 \\
-1 & 0 & 0 & 0 & \cdots & 0 & 0 & 0 & 0 \\
0 & -1 & 0 & 0 & \cdots & 0 & 0 & 0 & 0 \\
0 & 0 & -1 & 0 & \cdots & 0 & 0 & 0 & 0
\end{array}\right) .
\end{aligned}
$$

It can be checked that this matrix has the zero modes 


$$
\begin{aligned}
\mathbf{v}^{(3)}(x)= & \left(u, v, \omega, \chi, y, z, 0,0, m^{1} a_{1}^{-} \psi_{R}^{1}, m^{1} a_{1}^{+} \psi_{L}^{1}, m^{2} a_{2}^{-} \psi_{R}^{2}, m^{2} a_{2}^{+} \psi_{L}^{2}, \frac{m^{3}}{2} a_{3}^{-} \psi_{R}^{3},\right. \\
& \frac{m^{3}}{2} a_{3}^{+} \psi_{L}^{3}, u^{\prime}, v^{\prime}, i m^{1} a_{1}^{-} \widetilde{\psi}_{R}^{1}, i m^{1} a_{1}^{+} \widetilde{\psi}_{L}^{1}, i m^{2} a_{2}^{-} \widetilde{\psi}_{R}^{2}, i m^{2} a_{2}^{+} \widetilde{\psi}_{L}^{2}, \\
& \left.i \frac{m^{3}}{2} a_{3}^{-} \widetilde{\psi}_{R}^{3}, i \frac{m^{3}}{2} a_{3}^{+} \widetilde{\psi}_{L}^{3}\right),
\end{aligned}
$$

where $a_{1}^{+} \equiv u+\omega+y, a_{2}^{+} \equiv v+\chi+z, a_{3}^{+} \equiv u+\omega+y+v+\chi+z, a_{1}^{-} \equiv u+\omega-y, a_{2}^{-} \equiv v+\chi-z$, $a_{3}^{+} \equiv u+\omega+y+v-\chi-z$, and $u, v, \omega, \chi, y$, and $z$ are arbitrary functions. The relevant zero-mode condition gives no constraints. Then the action has the following symmetries:

$$
\delta \xi_{A}^{(3)}=\mathbf{v}_{\mathbf{A}}^{(3)}(x), \quad A=1,2, \ldots, 22 .
$$

These symmetries allow us to fix the bilinears $i \psi_{R}^{j} \psi_{L}^{j}$ to be constants. By taking $\psi_{R}^{j}$ $=-i C_{j} \bar{\theta}_{j}$ and $\psi_{L}^{j}=\theta_{j} \quad(j=1,2,3)$ with $C_{j}$ being real numbers, we find that $i \psi_{R}^{j} \psi_{L}^{j}$ indeed becomes a constant. Note that $\theta_{j}$ and $\bar{\theta}_{j}$ are Grassmannian variables, while $\bar{\theta}_{j} \theta_{j}$ is an ordinary commuting number.

The two form $f_{A B}^{(3)}(x, y)$, Eq. (4.36), in the subspace $\left(\phi_{1}, \phi_{2}, \pi_{\phi_{1}}, \pi_{\phi_{2}}\right)$ defines a canonical symplectic structure modulo canonical transformations. The coordinates $\phi_{a}$ and $\pi_{\phi_{a}}(a=1,2)$ are not unique. Consider a canonical transformation from $\left(\phi_{a}, \pi_{\phi_{a}}\right)$ to $\left(\hat{\phi}_{a}, \hat{\pi}_{\hat{\phi}_{a}}\right)$ such that $\phi_{a}$ $=\partial F / \partial \pi_{\phi_{a}}$ and $\hat{\phi}_{a}=\partial F / \partial \hat{\pi}_{\hat{\phi}_{a}}$. Then, in particular if $\phi_{a}=\hat{\phi}_{a}$ one can, in principle, solve for the function $F$ such that a manifestly covariant kinetic term appears in the new Lagrangian.

Then choosing $k=1 / g$ as the overall coupling constant, we are left with

$$
\mathcal{L}^{\prime \prime}=\sum_{j=1}^{3}\left[\frac{1}{24 g} \partial_{\mu} \phi_{j} \partial^{\mu} \phi_{j}+\frac{M_{j}}{g} \cos \phi_{j}\right]+\mu_{1} \partial_{x} \phi_{1}+\mu_{2} \partial_{x} \phi_{2},
$$

where $M_{j}=m_{\psi}^{j} C_{j}$. This defines the generalized sine-Gordon model (GSG). In addition we get the terms multiplied by chemical potentials $\mu_{1}$ and $\mu_{2}\left(\dot{\eta}^{1,2} \rightarrow-\mu_{1,2}\right)$. These are just the topological charge densities, and are related to the conservation of the number of kinks minus antikinks $Q_{\text {topol }}^{a}=1 / \pi \int_{-\infty}^{+\infty} \mathrm{d} x \partial_{x} \phi_{a}$.

In the above-mentioned gauge fixing procedures the possibility of Gribov-type ambiguities deserves a careful analysis. See Ref. 11 for a discussion in the $s l(2)$ ATM case. However, in Sec. $\mathrm{V}$, we provide indirect evidence of the absence of such ambiguities, at least for the soliton sector of the model.

\section{THE SOLITON/PARTICLE CORRESPONDENCES}

The $\operatorname{sl}(2)$ ATM theory contains the sine-Gordon (SG) and the massive Thirring (MT) models describing the soliton/particle correspondence of its spectrum. ${ }^{3,11,12}$ The ATM one-(anti)soliton solution satisfies the remarkable SG and MT classical correspondence in which, apart from the Noether and topological currents equivalence, MT spinor bilinears are related to the exponential of the SG field. ${ }^{25}$ The last relationship was exploited in Ref. 4 to decouple the $\operatorname{sl}(2)$ ATM equations of motion into the SG and MT ones. Here we provide a generalization of that correspondence to the $\operatorname{sl}(3)$ ATM case. In fact, consider the relationships

$$
\frac{\psi_{R}^{1} \widetilde{\psi}_{L}^{1}}{i}=-\frac{1}{4 \Delta}\left[\left(m_{\psi}^{1} p_{1}-m_{\psi}^{3} p_{4}-m_{\psi}^{2} p_{5}\right) e^{i\left(\varphi_{2}-2 \varphi_{1}\right)}+m_{\psi}^{2} p_{5} e^{3 i\left(\varphi_{2}-\varphi_{1}\right)}+m_{\psi}^{3} p_{4} e^{-3 i \varphi_{1}}-m_{\psi}^{1} p_{1}\right],
$$




$$
\begin{gathered}
\psi_{R}^{2} \widetilde{\psi}_{L}^{2}=-\frac{1}{4 \Delta}\left[\left(m_{\psi}^{2} p_{2}-m_{\psi}^{1} p_{5}-m_{\psi}^{3} p_{6}\right) e^{i\left(\varphi_{1}-2 \varphi_{2}\right)}+m_{\psi}^{1} p_{5} e^{3 i\left(\varphi_{1}-\varphi_{2}\right)}-m_{\psi}^{3} p_{6} e^{-3 i \varphi_{2}}-m_{\psi}^{2} p_{2}\right], \\
\frac{\widetilde{\psi}_{R}^{3} \psi_{L}^{3}}{i}=-\frac{1}{4 \Delta}\left[\left(m_{\psi}^{3} p_{3}-m_{\psi}^{1} p_{4}+m_{\psi}^{2} p_{6}\right) e^{i\left(\varphi_{1}+\varphi_{2}\right)}+m_{\psi}^{1} p_{4} e^{3 i \varphi_{1}}-m_{\psi}^{2} p_{6} e^{3 i \varphi_{2}}-m_{\psi}^{3} p_{3}\right],
\end{gathered}
$$

where $\Delta \equiv \bar{a}_{11} \bar{a}_{22} \bar{a}_{33}+2 \bar{a}_{12} \bar{a}_{23} \bar{a}_{13}-\bar{a}_{11}\left(\bar{a}_{23}\right)^{2}-\left(\bar{a}_{12}\right)^{2} \bar{a}_{33}-\left(\bar{a}_{13}\right)^{2} \bar{a}_{22} ; \quad p_{1} \equiv\left(\bar{a}_{23}\right)^{2}-\bar{a}_{22} \bar{a}_{33} ; \quad p_{2}$ $\equiv\left(\bar{a}_{13}\right)^{2}-\bar{a}_{11} \bar{a}_{33} ; \quad p_{3} \equiv\left(\bar{a}_{12}\right)^{2}-\bar{a}_{11} \bar{a}_{22} ; p_{4} \equiv \bar{a}_{12} \bar{a}_{23}-\bar{a}_{22} \bar{a}_{13} ; \quad p_{5} \equiv \bar{a}_{13} \bar{a}_{23}-\bar{a}_{12} \bar{a}_{33} ; \quad p_{6} \equiv \bar{a}_{11} \bar{a}_{23}$ $-\bar{a}_{12} \bar{a}_{13}$ and the $\bar{a}_{i j}$ 's being the current-current coupling constants of the GMT model (3.13). Relationships (5.1)-(5.3) supplied with the conditions (2.1)-(2.3) and conveniently substituted into Eqs. (A13) and (A15)-(A23) decouple the $s l(3)^{(1)}$ CATM equations into the GSG (4.39) and GMT (3.13) equations of motion, respectively.

Moreover, one can show that the GSG (4.39) $M_{j}$ parameters and the GMT (3.13) couplings $\bar{a}_{i j}$ are related by

$$
\begin{aligned}
& \frac{2 \Delta M_{1}}{g\left(m_{\psi}^{1}\right)^{2}}=\bar{a}_{22}\left(-\frac{m_{\psi}^{3}}{m_{\psi}^{1}} \bar{a}_{13}+\bar{a}_{33}\right)+\bar{a}_{23}\left(-\bar{a}_{23}+\frac{m_{\psi}^{3}}{m_{\psi}^{1}} \bar{a}_{12}\right), \\
& \frac{2 \Delta M_{2}}{g\left(m_{\psi}^{2}\right)^{2}}=\bar{a}_{11}\left(-\frac{m_{\psi}^{3}}{m_{\psi}^{2}} \bar{a}_{23}+\bar{a}_{33}\right)+\bar{a}_{13}\left(-\bar{a}_{13}+\frac{m_{\psi}^{3}}{m_{\psi}^{2}} \bar{a}_{12}\right), \\
& \frac{2 \Delta M_{3}}{g\left(m_{\psi}^{3}\right)^{2}}=-\frac{m_{\psi}^{1} m_{\psi}^{2}}{\left(m_{\psi}^{3}\right)^{2}}\left(\bar{a}_{12} \bar{a}_{33}-\bar{a}_{13} \bar{a}_{23}\right)-\bar{a}_{11} \bar{a}_{22}+\left(\bar{a}_{12}\right)^{2} .
\end{aligned}
$$

Various limiting cases of the relationships (5.1)-(5.3) and (5.4)-(5.6) are possible. First, let us consider

$$
\bar{a}_{j k} \rightarrow\left\{\begin{array}{l}
\infty \quad j=k \neq l \quad(\text { for a given } l) \\
\text { finite } \quad \text { other cases }
\end{array}\right.
$$

then one has

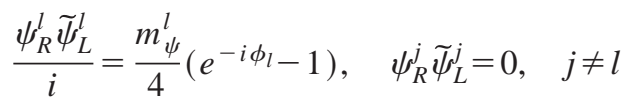

for $\bar{a}_{l l}=\delta_{l} g\left(\delta_{1,2}=1, \delta_{3}=-1\right)$. The three species of one-soliton solutions of the $\operatorname{sl}(3)$ ATM theory (2.4), found in Ref. 5 and described in Sec. II, satisfy the relationship (5.8). ${ }^{4}$ Moreover, from Eqs. (5.4) to (5.6) taking the same limits as in (5.7) one has

$$
M_{l}=\frac{\left(m_{\psi}^{l}\right)^{2}}{2}, \quad M_{j}=0, \quad j \neq l .
$$

Therefore, relationships (5.1)-(5.3) incorporate each $s l(2)$ ATM submodel (particle/soliton) weak/strong coupling phases, i.e., the MT/SG correspondence. ${ }^{4,11}$

Then, the currents equivalence (A29), relationships (5.1)-(5.3), and conditions (2.1)-(2.3) satisfied by the one-soliton sector of CATM theory allowed us to establish the correspondence between the GSG and GMT models, thus extending the MT/SG result. ${ }^{25}$ It could be interesting to obtain the counterpart of Eqs. (5.1)-(5.3) for the $N_{S} \geqslant 2$ solitons, e.g., along the lines of Ref. 25. For $N_{S}=2$, Eq. (A29) still holds; ${ }^{5}$ and Eqs. (2.1)-(2.3) are satisfied for the species $\left(\alpha_{i}, \alpha_{i}\right)$.

Second, consider the limit 


$$
\bar{a}_{i k} \rightarrow\left\{\begin{array}{l}
\infty \quad i=k=j \quad(\text { for a chosen } j ; j=1,2) \\
\text { finite other cases }
\end{array},\right.
$$

one gets $M_{j}=0$ and

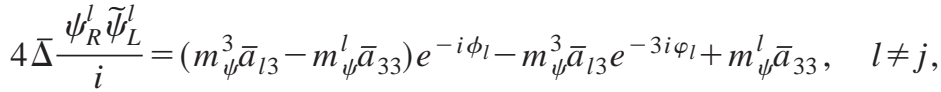

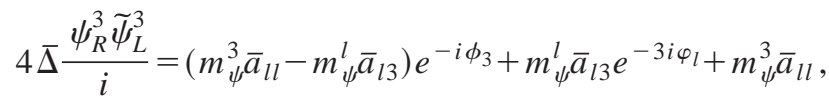

$$
\begin{aligned}
& \psi_{R}^{j} \widetilde{\psi}_{L}^{j}=0,
\end{aligned}
$$

where $\bar{\Delta} \equiv 4\left(\bar{a}_{l l} \bar{a}_{33}-\left(\bar{a}_{l 3}\right)^{2}\right)$. The parameters are related by $\left(m_{\psi}^{3}\right)^{2} \bar{a}_{l l} M_{l}=m_{\psi}^{l}\left(m_{\psi}^{3} \bar{a}_{l 3}\right.$ $\left.-m_{\psi}^{l} \bar{a}_{33}\right) M_{3}$. In the case $M_{l}=M_{3}=M$ and redefining the fields as $\phi_{l}=\sqrt{12 g}(A+B), \phi_{j}$ $=-\sqrt{12 g} B$ in the GSG sector, one gets the Lagrangian

$$
\mathcal{L}_{\mathrm{BL}}=\frac{1}{2}\left(\partial_{\mu} A\right)^{2}+\frac{1}{2}\left(\partial_{\mu} B\right)^{2}+2 \frac{M}{g} \cos \sqrt{24 g} A \cos \sqrt{72 g} B,
$$

which is a particular case of the Bukhvostov-Lipatov model (BL) ${ }^{26}$ It corresponds to a GMT-type theory with two Dirac spinors. The BL model is not classically integrable,${ }^{27}$ and some discussions have appeared in the literature about its quantum integrability. ${ }^{28}$

Alternatively, if one allows the limit $\bar{a}_{33} \rightarrow \infty$ one gets $\psi_{R}^{3} \widetilde{\psi}_{L}^{3}=0$, and additional relations for the $\psi^{1}, \psi^{2}$ spinors and the $\varphi_{a}$ scalars. The parameters are related by

$$
\frac{M_{1}}{\left(m_{\psi}^{1}\right)^{2} \bar{a}_{22}}=\frac{M_{2}}{\left(m_{\psi}^{2}\right)^{2} \bar{a}_{11}}=-\frac{M_{3}}{m_{\psi}^{1} m_{\psi}^{2} \bar{a}_{12}} .
$$

Then we left with two Dirac spinors in the GMT sector and all the terms of the GSG model. The later resembles the 2-cosine model studied in Ref. 29 in some submanifold of its renormalized parameter space.

\section{GENERALIZATION TO HIGHER RANK LIE ALGEBRA}

The procedures presented so far can directly be extended to the CATM model for the affine Lie algebra $s l(n)^{(1)}$ furnished with the principal gradation. According to the construction of Ref. 2 , these models have soliton solutions for an off-critical submodel, possess a $U(1)$ vector current proportional to a topological current, apart from the conformal symmetry they exhibit a $\left(U(1)_{R}\right)^{n-1} \otimes\left(U(1)_{L}\right)^{n-1}$ left-right local gauge symmetry, and the equations of motion describe the dynamics of the scalar fields $\varphi_{a}, \eta, \widetilde{\nu}(a=1, \ldots, n-1)$ and the Dirac spinors $\psi^{\alpha_{j}}, \widetilde{\psi}^{\alpha_{j}}(j$ $=1, \ldots, N ; N \equiv(n / 2)(n-1)=$ number of positive roots $\alpha_{j}$ of the simple Lie algebra $\left.s l(n)\right)$ with one-(anti)soliton solution associated with the field $\alpha_{j} \cdot \vec{\varphi}\left(\vec{\varphi}=\sum_{a=1}^{n-1} \varphi_{a} \alpha_{a}, \alpha_{a}=\right.$ simple roots of $s l(n))$ for each pair of Dirac fields $\left(\psi^{\alpha_{j}}, \widetilde{\psi}^{\alpha_{j}}\right) .{ }^{2}$ Therefore, it is possible to define the off-critical real Lagrangian $s l(n)$ ATM model for the solitonic sector of the theory. The reality conditions would generalize Eqs. (2.1)-(2.3), i.e., the new $\varphi$ 's real and the identifications $\widetilde{\psi}^{\alpha_{j}} \sim\left(\psi^{\alpha_{j}}\right)^{*}$ (up to \pm signs). To apply the symplectic analysis of $s l(n)$ ATM one must impose $(n-1)$ constraints in the Lagrangian, analogous to (3.1), due to the above-given local symmetries. The outcome will be a parent Lagrangian of a generalized massive Thirring model (GMT) with $N$ Dirac fields and a generalized sine-Gordon model (GSG) with $(n-1)$ fields. The decoupling of the Toda fields and Dirac fields in the equations of motion of $s l(n)^{(1)}$ CATM, analogous to (A13) and (A15)-(A23), could be performed by an extension of the relationships (5.1)-(5.3) and (2.1)-(2.3). 


\section{DISCUSSIONS AND OUTLOOK}

We have shown, in the context of FJ and symplectic methods, that the $s l(3)$ ATM (2.4) theory is a parent Lagrangian ${ }^{18}$ from which both the GMT (3.13) and the GSG (4.39) models are derivable. From (3.13) and (4.39), it is also clear the duality exchange of the couplings: $g \rightarrow 1 / g$. The various soliton/particle species correspondences are uncovered. The soliton sector satifies the $U(1)$ vector and topological currents equivalence (A29) and decouples the equations of motion into both dual sectors, through the relationships (5.1)-(5.3) [supplied with (2.1)-(2.3)]. Relationships (5.1)-(5.3) contain each $\operatorname{sl}(2)$ ATM submodel soliton solution. In connection to these points, recently a parent Lagrangian method was used to give a generalization of the dual theories concept for non- $p$-form fields. ${ }^{30}$ In Ref. 30 , the parent Lagrangian contained both types of fields, from which each dual theory was obtained by eliminating the other fields through the equations of motion.

On the other hand, in non-Abelian bosonization of massless fermions,${ }^{31}$ the fermion bilinears are identified with bosonic operators. Whereas, in Abelian bosonization ${ }^{32}$ there exists an identification between the massive fermion operator (charge nonzero sector) and a nonperturbative bosonic soliton operator. ${ }^{33}$ Recently, it has been shown that symmetric space sine-Gordon models bosonize the massive non-Abelian (free) fermions providing the relationships between the fermions and the relevant solitons of the bosonic model. ${ }^{34}$ The ATM model allowed us to establish these types of relationships for interacting massive spinors in the spirit of particle/soliton correspondence. We hope that the quantization of the ATM theories and the related WZNW models, and in particular relationships (A34), would provide the bosonization of the nonzero charge sectors of the GMT fermions in terms of their associated Toda and WZNW fields. In addition, the above-given approach to the GMT/GSG duality may be useful to construct the conserved currents and the algebra of their associated charges in the context of the CATM $\rightarrow$ ATM reduction. These currents in the MT/SG case were constructed treating each model as a perturbation on a conformal field theory (see Ref. 35 and references therein).

Moreover, two-dimensional models with four-fermion interactions have played an important role in the understanding of QCD (see, e.g., Ref. 36 and references therein). Besides, the GMT model contains explicit mass terms: most integrable models such as the Gross-Neveu, SU(2), and $\mathrm{U}(1)$ Thirring models all present spontaneous mass generation, the exception being the massive Thirring model. A GMT submodel with $a_{i i}=0, a_{i j}=1(i>j)$ and equal $m_{\psi}^{j}$ 's defines the so-called extended Bukhvostov-Lipatov model (BL) and has recently been studied by means of a bosonization technique. ${ }^{37}$ Finally, BL type models were applied to $N$-body problems in nuclear physics. ${ }^{17}$

\section{ACKNOWLEDGMENTS}

H.B. thanks Professor L. A. Ferreira and Professor A. H. Zimerman for discussions on integrable models and Professor M. B. Halpern and Professor R. K. Kaul for correspondences and valuable comments on the manuscript. The authors thank Professor B. M. Pimentel for discussions on constrained systems and Professor A. J. Accioly for encouragement. The authors are supported by FAPESP.

\section{APPENDIX: THE $s /(3)^{(1)}$ CATM MODEL}

We summarize the construction and some properties of the CATM model relevant to our discussions. ${ }^{38}$ More details can also be found in Ref. 5. Consider the zero curvature condition $\partial_{+} A_{-}-\partial_{-} A_{+}+\left[A_{+}, A_{-}\right]=0$. The potentials take the form

$$
A_{+}=-B F^{+} B^{-1}, \quad A_{-}=-\partial_{-} B B^{-1}+F^{-},
$$

with

$$
F^{+}=E^{3}+F_{1}^{+}+F_{2}^{+}, \quad F^{-}=E^{-3}+F_{1}^{-}+F_{2}^{-},
$$


where $E^{ \pm 3} \equiv \mathbf{m} \cdot H^{ \pm}=\frac{1}{6}\left[\left(2 m_{\psi}^{1}+m_{\psi}^{2}\right) H_{1}^{ \pm 1}+\left(2 m_{\psi}^{2}+m_{\psi}^{1}\right) H_{2}^{ \pm 1}\right]$ and the $F_{i}^{ \pm}$s and $B$ contain the spinor fields and scalars of the model, respectively,

$$
\begin{gathered}
F_{1}^{+}=\sqrt{i m_{\psi}^{1}} \psi_{R}^{1} E_{\alpha_{1}}^{0}+\sqrt{i m_{\psi}^{2}} \psi_{R}^{2} E_{\alpha_{2}}^{0}+\sqrt{i m_{\psi}^{3}} \widetilde{\psi}_{R}^{3} E_{-\alpha_{3}}^{1}, \\
F_{2}^{+}=\sqrt{i m_{\psi}^{3}} \psi_{R}^{3} E_{\alpha_{3}}^{0}+\sqrt{i m_{\psi}^{1}} \widetilde{\psi}_{R}^{1} E_{-\alpha_{1}}^{1}+\sqrt{i m_{\psi}^{2}} \widetilde{\psi}_{R}^{2} E_{-\alpha_{2}}^{1}, \\
F_{1}^{-}=\sqrt{i m_{\psi}^{3}} \psi_{L}^{3} E_{\alpha_{3}}^{-1}-\sqrt{i m_{\psi}^{1}} \widetilde{\psi}_{L}^{1} E_{-\alpha_{1}}^{0}-\sqrt{i m_{\psi}^{2}} \widetilde{\psi}_{L}^{2} E_{-\alpha_{2}}^{0}, \\
F_{2}^{-}=\sqrt{i m_{\psi}^{1}} \psi_{L}^{1} E_{\alpha_{1}}^{-1}+\sqrt{i m_{\psi}^{2}} \psi_{L}^{2} E_{\alpha_{2}}^{-1}-\sqrt{i m_{\psi}^{3}} \widetilde{\psi}_{L}^{3} E_{-\alpha_{3}}^{0}, \\
B=e^{i \varphi_{1} H_{1}^{0}+i \varphi_{2} H_{2}^{0}} e^{\widetilde{\nu} C} e^{\eta Q_{p p a l},}
\end{gathered}
$$

where $E_{\alpha_{i}}^{n}, H_{1}^{n}, H_{2}^{n}$, and $C(i=1,2,3 ; n=0, \pm 1)$ are some generators of $s l(3)^{(1)} ; Q_{p p a l}$ being the principal gradation operator. The commutation relations for an affine Lie algebra in the Chevalley basis are

$$
\begin{gathered}
{\left[H_{a}^{m}, H_{b}^{n}\right]=m C \frac{2}{\alpha_{a}^{2}} K_{a b} \delta_{m+n, 0},} \\
{\left[H_{a}^{m}, E_{ \pm \alpha}^{n}\right]= \pm K_{\alpha a} E_{ \pm \alpha}^{m+n},} \\
{\left[E_{\alpha}^{m}, E_{-\alpha}^{n}\right]=\sum_{a=1}^{r} l_{a}^{\alpha} H_{a}^{m+n}+\frac{2}{\alpha^{2}} m C \delta_{m+n, 0},} \\
{\left[E_{\alpha}^{m}, E_{\beta}^{n}\right]=\varepsilon(\alpha, \beta) E_{\alpha+\beta}^{m+n} ; \quad \text { if } \alpha+\beta \text { is a roo, }} \\
{\left[D, E_{\alpha}^{n}\right]=n E_{\alpha}^{n}, \quad\left[D, H_{a}^{n}\right]=n H_{a}^{n},}
\end{gathered}
$$

where $K_{\alpha a}=2 \alpha . \alpha_{a} / \alpha_{a}^{2}=n_{b}^{\alpha} K_{b a}$, with $n_{a}^{\alpha}$ and $l_{a}^{\alpha}$ being the integers in the expansions $\alpha=n_{a}^{\alpha} \alpha_{a}$ and $\alpha / \alpha^{2}=l_{a}^{\alpha} \alpha_{a} / \alpha_{a}^{2}$, and $\varepsilon(\alpha, \beta)$ the relevant structure constants.

Take $K_{11}=K_{22}=2$ and $K_{12}=K_{21}=-1$ as the Cartan matrix elements of the simple Lie algebra $\operatorname{sl}(3)$. Denoting by $\alpha_{1}$ and $\alpha_{2}$ the simple roots and the highest one by $\psi\left(=\alpha_{1}+\alpha_{2}\right)$, one has $l_{a}^{\psi}=1(a=1,2)$, and $K_{\psi 1}=K_{\psi 2}=1$. Take $\varepsilon(\alpha, \beta)=-\varepsilon(-\alpha,-\beta), \varepsilon_{1,2} \equiv \varepsilon\left(\alpha_{1}, \alpha_{2}\right)=1, \varepsilon_{-1,3}$ $\equiv \varepsilon\left(-\alpha_{1}, \psi\right)=1$ and $\varepsilon_{-2,3} \equiv \varepsilon\left(-\alpha_{2}, \psi\right)=-1$.

One has $Q_{\text {ppal }} \equiv \Sigma_{a=1}^{2} \mathbf{s}_{a} \lambda_{a}^{v} \cdot H+3 D$, where $\lambda_{a}^{v}$ are the fundamental co-weights of $s l(3)$, and the principal gradation vector is $\mathbf{s}=(1,1,1) .{ }^{39}$

The zero curvature condition gives the following equations of motion

$$
\begin{gathered}
\frac{\partial^{2} \varphi_{a}}{4 i e^{\eta}}=m_{\psi}^{1}\left[e^{\eta-i \phi_{a}} \widetilde{\psi}_{R}^{l} \psi_{L}^{l}+e^{i \phi_{a}} \widetilde{\psi}_{L}^{l} \psi_{R}^{l}\right]+m_{\psi}^{3}\left[e^{-i \phi_{3}} \widetilde{\psi}_{R}^{3} \psi_{L}^{3}+e^{\eta+i \phi_{3}} \widetilde{\psi}_{L}^{3} \psi_{R}^{3}\right], \quad a=1,2, \\
-\frac{\partial^{2} \widetilde{v}}{4}=i m_{\psi}^{1} e^{2 \eta-\phi_{1}} \widetilde{\psi}_{R}^{1} \psi_{L}^{1}+i m_{\psi}^{2} e^{2 \eta-\phi_{2}} \widetilde{\psi}_{R}^{2} \psi_{L}^{2}+i m_{\psi}^{3} e^{\eta-\phi_{3}} \widetilde{\psi}_{R}^{3} \psi_{L}^{3}+\mathbf{m}^{2} e^{3 \eta} \\
-2 \partial_{+} \psi_{L}^{1}=m_{\psi}^{1} e^{\eta+i \phi_{1}} \psi_{R}^{1}, \quad-2 \partial_{+} \psi_{L}^{2}=m_{\psi}^{2} e^{\eta+i \phi_{2}} \psi_{R}^{2} \\
2 \partial_{-} \psi_{R}^{1}=m_{\psi}^{1} e^{2 \eta-i \phi_{1}} \psi_{L}^{1}+2 i\left(\frac{m_{\psi}^{2} m_{\psi}^{3}}{i m_{\psi}^{1}}\right)^{1 / 2} e^{\eta}\left(-\psi_{R}^{3} \widetilde{\psi}_{L}^{2} e^{i \phi_{2}-} \widetilde{\psi}_{R}^{2} \psi_{L}^{3} e^{-i \phi_{3}}\right)
\end{gathered}
$$




$$
\begin{aligned}
& 2 \partial_{-} \psi_{R}^{2}=m_{\psi}^{2} e^{2 \eta-i \phi_{2}} \psi_{L}^{2}+2 i\left(\frac{m_{\psi}^{1} m_{\psi}^{3}}{i m_{\psi}^{2}}\right)^{1 / 2} e^{\eta}\left(\psi_{R}^{3} \widetilde{\psi}_{L}^{1} e^{i \phi_{1}}+\widetilde{\psi}_{R}^{1} \psi_{L}^{3} e^{-i \phi_{3}}\right), \\
& -2 \partial_{+} \psi_{L}^{3}=m_{\psi}^{3} e^{2 \eta+i \phi_{3}} \psi_{R}^{3}+2 i\left(\frac{m_{\psi}^{1} m_{\psi}^{2}}{i m_{\psi}^{3}}\right)^{1 / 2} e^{\eta}\left(-\psi_{L}^{1} \psi_{R}^{2} e^{i \phi_{2}}+\psi_{L}^{2} \psi_{R}^{1} e^{i \phi_{1}}\right) \\
& 2 \partial_{-} \psi_{R}^{3}=m_{\psi}^{3} e^{\eta-i \phi_{3}} \psi_{L}^{3}, \quad 2 \partial_{-} \widetilde{\psi}_{R}^{1}=m_{\psi}^{1} e^{\eta+i \phi_{1}} \widetilde{\psi}_{L}^{1}, \\
& -2 \partial_{+} \widetilde{\psi}_{L}^{1}=m_{\psi}^{1} e^{2 \eta-i \phi_{1}} \widetilde{\psi}_{R}^{1}+2 i\left(\frac{m_{\psi}^{2} m_{\psi}^{3}}{i m_{\psi}^{1}}\right)^{1 / 2} e^{\eta}\left(-\psi_{L}^{2} \widetilde{\psi}_{R}^{3} e^{-i \phi_{3}}-\widetilde{\psi}_{L}^{3} \psi_{R}^{2} e^{i \phi_{2}}\right), \\
& -2 \partial_{+} \widetilde{\psi}_{L}^{2}=m_{\psi}^{2} e^{2 \eta-i \phi_{2}} \widetilde{\psi}_{R}^{2}+2 i\left(\frac{m_{\psi}^{1} m_{\psi}^{3}}{i m_{\psi}^{2}}\right)^{1 / 2} e^{\eta}\left(\psi_{L}^{1} \widetilde{\psi}_{R}^{3} e^{-i \phi_{3}}+\widetilde{\psi}_{L}^{3} \psi_{R}^{1} e^{i \phi_{1}}\right), \\
& 2 \partial_{-} \widetilde{\psi}_{R}^{2}=m_{\psi}^{2} e^{\eta+i \phi_{2}} \widetilde{\psi}_{L}^{2}, \quad-2 \partial_{+} \widetilde{\psi}_{L}^{3}=m_{\psi}^{3} e^{\eta-i \phi_{3}} \widetilde{\psi}_{R}^{3}, \\
& 2 \partial_{-} \widetilde{\psi}_{R}^{3}=m_{\psi}^{3} e^{2 \eta+i \phi_{3}} \widetilde{\psi}_{L}^{3}+2 i\left(\frac{m_{\psi}^{1} m_{\psi}^{2}}{i m_{\psi}^{3}}\right)^{1 / 2} e^{\eta}\left(\widetilde{\psi}_{R}^{1} \widetilde{\psi}_{L}^{2} e^{i \phi_{2}}-\widetilde{\psi}_{R}^{2} \widetilde{\psi}_{L}^{1} e^{i \phi_{1}}\right), \\
& \partial^{2} \eta=0,
\end{aligned}
$$

where $\phi_{1} \equiv 2 \varphi_{1}-\varphi_{2}, \phi_{2} \equiv 2 \varphi_{2}-\varphi_{1}, \phi_{3} \equiv \varphi_{1}+\varphi_{2}$.

Apart from the conformal invariance the above-presented equations exhibit the $\left(U(1)_{L}\right)^{2}$ $\otimes\left(U(1)_{R}\right)^{2}$ left-right local gauge symmetry

$$
\begin{gathered}
\varphi_{a} \rightarrow \varphi_{a}+\theta_{+}^{a}\left(x_{+}\right)+\theta_{-}^{a}\left(x_{-}\right), \quad a=1,2, \\
\widetilde{\nu} \rightarrow \widetilde{\nu}, \quad \eta \rightarrow \eta, \\
\psi^{i} \rightarrow e^{i\left(1+\gamma_{5}\right) \Theta_{+}^{i}\left(x_{+}\right)+i\left(1-\gamma_{5}\right) \Theta_{-}^{i}\left(x_{-}\right)} \psi^{i}, \\
\widetilde{\psi}^{i} \rightarrow e^{-i\left(1+\gamma_{5}\right)\left(\Theta_{+}^{i}\right)\left(x_{+}\right)-i\left(1-\gamma_{5}\right)\left(\Theta_{-}^{i}\right)\left(x_{-}\right)} \widetilde{\psi}^{i}, \quad i=1,2,3, \\
\Theta_{ \pm}^{1} \equiv \pm \theta_{ \pm}^{2} \mp 2 \theta_{ \pm}^{1}, \quad \Theta_{ \pm}^{2} \equiv \pm \theta_{ \pm}^{1} \mp 2 \theta_{ \pm}^{2}, \quad \Theta_{ \pm}^{3} \equiv \Theta_{ \pm}^{1}+\Theta_{ \pm}^{2} .
\end{gathered}
$$

One can get global symmetries for $\theta_{ \pm}^{a}=\mp \theta_{\mp}^{a}=$ constants. For a model defined by a Lagrangian these would imply the presence of two vector and two chiral conserved currents. However, it was found only half of such currents. ${ }^{5}$ This is a consequence of the lack of a Lagrangian description for the $\operatorname{sl}(3)^{(1)}$ CATM; however see the following.

The gauge fixing of the conformal symmetry, by setting the field $\eta$ to a constant, is used to stablish the $U(1)$ vector, $J^{\mu}=\Sigma_{j=1}^{3} m_{\psi}^{j} \bar{\psi}^{j} \gamma^{\mu} \psi^{j}$, and topological currents equivalence. ${ }^{2,11}$ Moreover, it has been shown that the soliton solutions are in the orbit of the solution $\eta=0$. The remarkable equivalence is

$$
\sum_{j=1}^{3} m_{\psi}^{j} \bar{\psi}^{j} \gamma^{\mu} \psi^{j} \equiv \epsilon^{\mu \nu} \partial_{\nu}\left(m_{\psi}^{1} \varphi_{1}+m_{\psi}^{2} \varphi_{2}\right), \quad m_{\psi}^{3}=m_{\psi}^{1}+m_{\psi}^{2}, \quad m_{\psi}^{i}>0 .
$$

The CATM theory has a local Lagrangian in terms of the $B$ and the (two-loop) WZNW fields. ${ }^{2}$ The relations between their fields can be obtained from

$$
F^{-}=B \partial_{-} M M^{-1} B^{-1}, \quad F^{+}=B^{-1} N^{-1} \partial_{+} N B,
$$


where

$$
M=\exp \left(\sum_{s>0} \zeta_{s}\right), \quad N=\exp \left(\sum_{s>0} \xi_{-s}\right)
$$

provided that the following constraints are imposed:

$$
\left(\partial_{-} M M^{-1}\right)_{-3}=B^{-1}\left(\mathbf{m} \cdot H^{-1}\right) B, \quad\left(\partial_{-} M M^{-1}\right)_{<-3}=0 .
$$

and

$$
\left(N^{-1} \partial_{+} N\right)_{3}=B\left(\mathbf{m} \cdot H^{1}\right) B^{-1}, \quad\left(N^{-1} \partial_{+} N\right)_{>3}=0 .
$$

In (A31) and (A32) and (A33) $s$ and the subscripts denote the principal gradation structure of the relevant group elements.

The relationships are

$$
\begin{aligned}
& \sqrt{i m_{\psi}^{3}} \psi_{L}^{3}=e^{-\eta+i \phi_{3}} \partial_{-} \xi_{-1}^{3}, \quad-\sqrt{i m_{\psi}^{1}} \widetilde{\psi}_{L}^{1}=e^{-\eta-i \phi_{1}} \partial_{-} \xi_{-1}^{1}, \\
& -\sqrt{i m_{\psi}^{2}} \widetilde{\psi}_{L}^{2}=e^{-\eta-i \phi_{2}} \partial_{-} \xi_{-1}^{2}, \quad \sqrt{i m_{\psi}^{3}} \psi_{R}^{1}=e^{\eta-i \phi_{1}} \partial_{+} \zeta_{1}^{1}, \\
& \sqrt{i m_{\psi}^{2}} \psi_{R}^{2}=e^{\eta-i \phi_{2}} \partial_{+} \zeta_{1}^{2}, \quad \sqrt{i m_{\psi}^{3}} \widetilde{\psi}_{R}^{3}=e^{\eta+i \phi_{3}} \partial_{+} \zeta_{1}^{3}, \\
& \sqrt{i m_{\psi}^{1}} \psi_{L}^{1} e^{2 \eta}=e^{i \phi_{1}} \partial_{-} \xi_{-2}^{1}+\frac{1}{2}\left(\xi_{-1}^{3} \partial_{-} \xi_{-1}^{2} \epsilon_{3,-2}+\xi_{-1}^{2} \partial_{-} \xi_{-1}^{3} \epsilon_{-2,3}\right) e^{i \phi_{1}}, \\
& \sqrt{i m_{\psi}^{2}} \psi_{L}^{2} e^{2 \eta}=e^{i \phi_{2}} \partial_{-} \xi_{-2}^{2}+\frac{1}{2}\left(\xi_{-1}^{3} \partial_{-} \xi_{-1}^{1} \epsilon_{3,-1}+\xi_{-1}^{1} \partial_{-} \xi_{-1}^{3} \epsilon_{-1,2}\right) e^{i \phi_{2}}, \\
& -\sqrt{i m_{\psi}^{3}} \widetilde{\psi}_{L}^{3} e^{2 \eta}=e^{-i \phi_{3}} \partial_{-} \xi_{-2}^{3}+\frac{1}{2}\left(\xi_{-1}^{1} \partial_{-} \xi_{-1}^{2} \epsilon_{-1,-2}+\xi_{-1}^{2} \partial_{-} \xi_{-1}^{1} \epsilon_{-2,-1}\right) e^{-i \phi_{3}}, \\
& \sqrt{i m_{\psi}^{3}} \psi_{R}^{3} e^{-2 \eta}=e^{-i \phi_{3}} \partial_{+} \zeta_{2}^{3}-\frac{1}{2}\left(\zeta_{1}^{1} \partial_{+} \zeta_{1}^{2} \epsilon_{1,2}+\zeta_{1}^{2} \partial_{+} \zeta_{1}^{1} \epsilon_{2,1}\right) e^{-i \phi_{3}}, \\
& \sqrt{i m_{\psi}^{1}} \widetilde{\psi}_{R}^{1} e^{-2 \eta}=e^{i \phi_{1}} \partial_{+} \zeta_{2}^{1}-\frac{1}{2}\left(\zeta_{1}^{3} \partial_{+} \zeta_{1}^{2} \epsilon_{-3,2}+\zeta_{1}^{2} \partial_{+} \zeta_{1}^{3} \epsilon_{2,-3}\right) e^{i \phi_{1}}, \\
& \sqrt{i m_{\psi}^{2}} \widetilde{\psi}_{R}^{2} e^{-2 \eta}=e^{i \phi_{2}} \partial_{+} \zeta_{2}^{2}-\frac{1}{2}\left(\zeta_{1}^{3} \partial_{+} \zeta_{1}^{1} \epsilon_{-3,1}+\zeta_{1}^{1} \partial_{+} \zeta_{1}^{3} \epsilon_{1,-3}\right) e^{i \phi_{2}}
\end{aligned}
$$

We observe that the WZNW fields $\xi_{-1}^{i}, \xi_{-2}^{i}, \zeta_{1}^{i}, \zeta_{2}^{i}(i=1,2,3)$ are nonlocal in terms of the spinors and scalars $\left\{\psi_{i}, \widetilde{\psi}_{i}, \varphi_{1}, \varphi_{2}, \widetilde{\nu}\right.$, and $\left.\eta\right\}$. Then the CATM model Lagrangian must be nonlocal when written in terms of its fields.

${ }^{1}$ E. Abdalla, M. C. B. Abdalla, and K. D. Rothe, Non-perturvative Methods in Two-dimensional Quantum Field Theory (World Scientific, Singapore, 1991).

${ }^{2}$ L. A. Ferreira, J-L. Gervais, J. Sánchez Guillen, and M. V. Saveliev, Nucl. Phys. B 470, 236 (1996).

${ }^{3}$ H. Blas and L. A. Ferreira, Nucl. Phys. B 571, 607 (2000).

${ }^{4}$ H. Blas, Nucl. Phys. B 596, 471 (2001); Proceedings of the seventh Hadron Physics 2000 Workshop, Caraguatatuba, SP, Brazil, April 2000, pp. 10-15 hep-th/0005037.

${ }^{5}$ A. G. Bueno, L. A. Ferreira, and A. V. Razumov, hep-th/0105078.

${ }^{6}$ C. Montonen and D. I. Olive, Phys. Lett. 72B, 117 (1977); P. Goddard, J. Nuyts, and D .I. Olive, Nucl. Phys. B 125, 1 (1977).

${ }^{7}$ C. Vafa and E. Witten, Nucl. Phys. B 431, 3 (1994); N. Seiberg and E. Witten, ibid. 431, 484 (1994); 426, 19 (1994); A. Sen, Phys. Lett. B 329, 217 (1994); Int. J. Mod. Phys. A 9, 3707 (1994).

${ }^{8}$ S. Brazovskii, J. Phys. IV 10, 169 (2000); also in cond-mat/0006355; A. J. Heeger, S. Kivelson, J. R. Schrieffer, and W.-P. Wu, Rev. Mod. Phys. 60, 781 (1988).

${ }^{9}$ D. G. Barci and L. Moriconi, Nucl. Phys. B 438, 522 (1995).

${ }^{10}$ R. Jackiw and C. Rebbi, Phys. Rev. D 13, 3398 (1976); J. Goldstone and F. Wilczek, Phys. Rev. Lett. 47, 986 (1981); J. A. Mignaco and M. A. Rego Monteiro, Phys. Rev. D 31, 3251 (1985).

${ }^{11}$ H. Blas and B. M. Pimentel, Ann. Phys. (Leipzig) 282, 67 (2000). 
${ }^{12}$ E. Witten, Nucl. Phys. B 145, 110 (1978).

${ }^{13}$ L. Faddeev and R. Jackiw, Phys. Rev. Lett. 60, 1692 (1988); R. Jackiw, Diverse Topics in Theoretical Physics, 1st ed. (World Scientific, Singapore, 1995).

${ }^{14}$ C. Wotzasek, Ann. Phys. (Leipzig) 243, 76 (1995); H. Montani and C. Wotzasek, Mod. Phys. Lett. A 8, 3387 (1993); J. Barcelos-Neto and C. Wotzasek, ibid. 7, 1737 (1992); Int. J. Mod. Phys. A 7, 4981 (1992).

${ }^{15}$ H. Montani, Int. J. Mod. Phys. A 8, 4319 (1993).

${ }^{16}$ H. Saleur and P. Simonetti, Nucl. Phys. B 535, 596 (1998).

${ }^{17}$ J. Sakamoto and Y. Heike, Prog. Theor. Phys. 100, 399 (1998); L. V. Belvedere and R. L. P. G. Amaral, Phys. Rev. D 100, 399 (1998).

${ }^{18}$ S. E. Hjelmeland and U. Lindström, hep-th/9705122.

${ }^{19}$ M. B. Halpern, Phys. Rev. D 12, 1684 (1975).

${ }^{20}$ Z. Zhu and D. G. Caldi, J. Math. Phys. 36, 6862 (1995); G. Takacs and G. Watts, Nucl. Phys. B 547, 538 (1999); S. P. Khastgir and R. Sasaki, Prog. Theor. Phys. 95, 485 (1996).

${ }^{21}$ The auxiliary fields $\widetilde{\nu}$ and $\eta$ of the CATM theories are associated with the topological character of the soliton masses and to the conformal symmetry, respectively. The classical and quantum reductions CATM $\rightarrow$ ATM can be treated as in Refs. 11 and 4, respectively.

${ }^{22} \operatorname{sl}(2)$ ATM 2-solitons satisfy an analogous equation to (A29); for $\varphi$ real and $\widetilde{\psi}= \pm(\psi) *$ constraints $(2.1)-(2.2)$; (2.3) is trivially satisfied since $\widetilde{\psi}_{j}=\psi_{j}=0$ for $\left.j \neq i\right]$ one has, soliton-soliton SS, SS bounds, and no S $\bar{S}$ bounds (Ref. 3).

${ }^{23}$ W. Siegel, Nucl. Phys. B 238, 307 (1984); M. Henneaux, Proceedings of the Summer School in High-Energy Physics and Cosmology (ICTP, Trieste, 1988)

${ }^{24}$ These are given for point mechanics, the extension to field theory is self-evident.

${ }^{25}$ S. J. Orfanidis and R. Wang, Phys. Lett. 57B, 281 (1975); S. J. Orfanidis, Phys. Rev. D 14, 472 (1976).

${ }^{26}$ A. P. Bukhvostov and L. N. Lipatov, Nucl. Phys. B 180, 116 (1981).

${ }^{27}$ M. Ameduri and C. J. Efthimiou, J. Nonlinear Math. Phys. 5, 132 (1998).

${ }^{28}$ H. Saleur, J. Phys. A 32, 207 (1999); M. Ameduri, C. J. Efthimiou, and B. Gerganov, Mod. Phys. Lett. A 14, 2341 (1999).

${ }^{29}$ B. Gerganov, Nucl. Phys. B 567, 391 (2000).

${ }^{30}$ H. Casini, R. Montemayor, and L. F. Urrutia, Phys. Lett. B 507, 336 (2001).

${ }^{31}$ E. Witten, Commun. Math. Phys. 92, 455 (1984).

${ }^{32}$ S. Coleman, Phys. Rev. D 11, 2088 (1975).

${ }^{33}$ S. Mandelstam, Phys. Rev. D 11, 3026 (1975).

${ }^{34}$ Q.-Han Park and H. J. Shin, Nucl. Phys. B 506, 537 (1997).

${ }^{35}$ R. K. Kaul and R. Rajaraman, Int. J. Mod. Phys. A 8, 1815 (1993).

${ }^{36}$ J. F. Bennett and J. A. Gracey, Nucl. Phys. B 563, 390 (1999).

${ }^{37}$ J. Sakamoto and B. R. Poudel, Prog. Theor. Phys. 104, 237 (2000).

${ }^{38}$ Our notations follow that of Ref. 2, except that the $\varphi$ 's below have been multiplied by $i$.

${ }^{39}$ V. G. Kac, Infinite Dimensional Lie Algebras, 3rd ed. (Cambridge University Press, Cambridge, 1990). 